\title{
LA COMISIÓN PARA LA CONSOLIDACIÓN DE LA PAZ EN GUINEA-BISÁU \\ O DE LOS LÍMITES DE LA PAZ INSTAURADA
}

\author{
THE PEACEBUILDING COMMISSION \\ IN GUINEA-BISSAU \\ OR THE LIMITS OF THE ESTABLISHED PEACE
}

\author{
LA COMMISSION DE CONSOLIDATION \\ DE LA PAIX EN GUINÉE-BISSAU \\ OU LES LIMITES DE LA PAIX INSTAURÉE
}

\author{
Thaíse Kemer \\ Universidad Federal de Paraná \\ thaise.kemer@gmail.com \\ Alexsandro Eugenio Pereira \\ Universidad Federal de Paraná \\ alexsep@uol.com.br \\ RAMON BLANCO \\ Universidad Federal de la Integración Latinoamericana \\ blanco.ramon@gmail.com
}

Resumen: A partir de un estudio de caso y de investigación de archivo, este artículo discute las prácticas de la Comisión para la Consolidación de la Paz de las Naciones Unidas (PвC, según sus siglas en inglés) en Guinea-Bisáu, con el propósito de replantear el concepto de Consolidación de la paz que surge de la actuación de la Comisión. El argumento esencial consiste en que la PBC, al presuponer la validez universal del paradigma de la paz liberal, no considera adecuadamente los condicionantes históricos, políticos y socioeconómicos locales. Al señalar una inadecuación entre la PBC y la realidad local, este estudio amplía el debate sobre el papel de la PBC en escenarios posteriores a los conflictos contemporáneos.

Palabras clave: Naciones Unidas; consolidación de la paz; Guinea-Bisáu; desarrollo; apropiación local. 
Abstract: Based on a case study and archival research, this article discusses the practices of the UN Peacebuilding Commission ( $\mathrm{PBC}$ ) in Guinea-Bissau, with the aim of rethinking the concept of Peace consolidation that arises from the acts of the Commission. The essential argument is that the PBC, by assuming the universal validity of the liberal peace paradigm, fails to adequately consider the local historical, political and socioeconomic conditions. By noting the poor fit between the $\mathrm{PBC}$ approach and the local reality, this study expands the debate on the role of the $\mathrm{PBC}$ in scenarios following the contemporary conflicts.

Keywords: United Nations; peacebuilding; Guinea-Bissau; development; local appropriation.

\section{Traducción de Gonzalo Celorio Morayta}

RésumÉ: À partir de l'étude d'un cas particulier, appuyée sur des recherches en archives, l'article traite des activités de la Commission de consolidation de la paix des Nations Unies (PBC en anglais) en Guinée-Bissau, dans le but de remettre en cause le concept de Consolidation de la paix découlant des activités de cette Commission. À notre avis, la PBC, du fait qu'elle présuppose la validité universelle du modèle de la paix libérale, n'accorde pas assez d'attention aux conditions historiques, politiques et socio-économiques locales. Nous souhaitons que nos remarques sur cette défaillance puissent contribuer au débat concernant le rôle de la PBC dans des régions marquées par les conflits contemporains.

Mots clefs: Nations Unies; consolidation de la paix; Guinée-Bissau; développement; appropriation locale.

\section{Traducción de BERnARDo MABIRE}

Fecha de recepción: enero de 2018

Fecha de aceptación: julio de 2018 
A L observar la realidad internacional, puede percibirse que una de sus dinámicas fundamentales es la superación de los conflictos violentos. En este artículo, por tanto, se reflexiona sobre un actor importante en el asunto, la Comisión para la Consolidación de la Paz (PBC, en adelante) de las Naciones Unidas. Establecida en 2005, la PBC busca coordinar diferentes actores e iniciativas para apoyar los procesos de consolidación de la paz en países que intentan salir de los conflictos que padecen. En este artículo, por medio de la discusión crítica, se pondera su caso más emblemático: el método de participación con que la PBC intervino en GuineaBisáu. El propósito del análisis es comprender la actuación de la Comisión y debatir los cuestionamientos a la consolidación de la paz que surgen de ese entorno. ${ }^{1}$ Para ello, según adelantábamos en el resumen de este trabajo, se utilizan como instrumentos metodológicos el estudio de caso y la investigación de archivo. En cuanto al primero, la selección de Guinea-Bisáu se basó en una elección del tipo most likely. Los casos correspondientes a esta especie deberían producir un determinado fenómeno, pero nunca llegan a producirlo. Por lo tanto, se utilizan para refutar teorías y generar hipótesis. ${ }^{2}$ El proceso de selección de caso se basó en la elección del país con mayor Índice de Desarrollo Humano $(\mathrm{IDH})^{3}$ entre

${ }^{1}$ Sobre los reflejos de la paz liberal en la actuación de las Naciones Unidas en la consolidación de la paz en Guinea-Bisáu, véase F. CAvalcanTE, "Os reflexos da paz liberal na abordagem das Nações Unidas à consolidação da paz na Guiné-Bissau”, Revista Crítica de Ciências Sociais, 2013, núm. 102, pp. 21-42.

${ }^{2}$ R. Perissinotto, "Comparação, história e interpretação: Por uma ciência política histórico-interpretativa”, Revista Brasileira de Ciências Sociais, 28 (2013), núm. 83, p. 156.

${ }^{3}$ El Índice de Desarrollo Humano evalúa el progreso a largo plazo de tres dimensiones del desarrollo humano: una vida larga y saludable, el acceso al conocimiento y un patrón decente de vida. Su valor varía entre 0 y 1; este último indica que hay mejores condiciones de desarrollo. Véase PNUD, "Explanatory Note on the 2014 Human Development Report Composite Indices Guinea-Bissau”, 2014, p. 1, en http://hdr.undp.org/sites/ all/themes/hdr_theme/country-notes/GNB.pdf, consultado el 24.XI.2015. 
los países en la agenda de la PBC, el cual constituiría el "caso más probable" de éxito para la PBC en su intento por promover la consolidación de la paz. Considerando que en 2013 Guinea-Bisáu presentó uno de los valores más prominentes para el IDH entre los seis países en la $\mathrm{PBC},{ }^{4}$ se decidió escogerla para el estudio de caso. Respecto del segundo instrumento, el artículo examina documentos relevantes, como informes de reuniones, misiones y declaraciones, tanto de las Naciones Unidas cuanto del gobierno de Guinea-Bisáu y de organizaciones no gubernamentales.

El artículo se divide en tres apartados. En el primero, se presenta la forma de funcionamiento de la $\mathrm{PBC}$ y se discute el concepto de consolidación de la paz liberal que orienta sus actividades. En el segundo, se ofrece un panorama de Guinea-Bisáu, entre 2007 y 2014, en los ámbitos de la política, el desarrollo y la seguridad. El año de 2007 constituye el inicio de las actividades de la $\mathrm{PBC}$ en el país, en tanto que el de 2014 supone la celebración de las primeras elecciones democráticas tras el golpe militar de 2012. Estos tres ejes analíticos permiten ponderar de manera más amplia la realidad de Guinea-Bisáu, pues se relacionan con los objetivos de la PBC para ese país. En particular, en lo que hace a la seguridad, se adoptó como recorte temático la Reforma del Sector de Seguridad (RSs), la cual implica un proceso liderado por autoridades nacionales que tienen por propósito mejorar la efectividad de la seguridad nacional. ${ }^{5}$ Este recorte se justifica tanto por el hecho de que el gobierno haya adoptado una política específica para el tratamiento de este asunto $^{6}$ como por el de haber sido considerado tema priori-

${ }^{4}$ Idem.

${ }^{5}$ Naciones Unidas, "The United Nations ssR Perspective”, 2012, p. 2, en http://www.un.org/en/events/peacekeepersday/pdf/securityreform. pdf, consultado el 30.VIII.2015.

${ }^{6}$ República da Guiné-Bissau, "Reestructuring and Modernization of the Defence and Security Sector", 2006, en http://uniogbis.unmissions. org/Portals/unogbis/Articles/SSR\%20srtategy\%20document\%202006. pdf, consultado el 9.I.2016. 
tario para la consolidación de la paz en Guinea-Bisáu por la PBC. ${ }^{7}$ En el tercer apartado, las prácticas de la PBC se examinan a partir del ámbito guineano, para evidenciar que el proceso de consolidación de la paz en Guinea-Bisáu ofrece margen al cuestionamiento. El análisis ofrece pruebas empíricas que demuestran una desproporción entre los procedimientos de la PBC y la realidad local guineana y propone, además, un debate más amplio acerca del papel que desempeña la $\mathrm{PBC}$ en escenarios que se erigen tras los conflictos contemporáneos.

El CONCEPTO DE CONSOLIDACIÓN DE LA PAZ Y EL ESTABLECIMIENTO DE LA COMISIÓN para la Consolidación de la Paz DE LAS NaGiones Unidas

El concepto de consolidación de la paz $^{8}$ surgió en las Naciones Unidas en 1992 con el informe "Una agenda para la paz”, del Secretario general Boutros Boutros-Ghali. ${ }^{9}$ Este concepto retomó las ideas de Johan Galtung, quien interpretó la noción de paz no sólo como una "paz negativa", entendida como la ausencia de violencia directa, sino también como una "paz positiva", la cual, al relacionarse con la satisfacción de las necesidades humanas básicas, salud y

${ }^{7} \mathrm{PBC} / 4 / \mathrm{GNB} / 1$, "Progress Report on the Implementation of the Peacebuilding Strategic Framework for Guinea-Bissau”, 2009, § 49, en http:/ / www.un.org/ga/search/view_doc.asp?symbol=PBC/4/GNB/1, consultado el 17.I.2016.

${ }^{8}$ Una discusión sobre el concepto de consolidación de la paz, como sobre las críticas a este concepto, puede encontrarse en T. Kemer et al., "A construção da paz em um mundo em transformação: o debate e a crítica sobre o conceito de peacebuilding", Revista de Sociologia e Política, 24 (2016), núm. 60, pp. 137-150.

9 A/47/277, “An Agenda for Peace”, 17 de junio de 1992, en http:/ / daccess-dds-y.un.org/doc/UNDOC/GEN/N92/259/61/PDF/N9225961. pdf?OpenElement, consultado el 20.VIII.2014. 
educación, por ejemplo, permitiría el desarrollo pleno de las capacidades humanas. ${ }^{10}$

La consolidación de la paz fue parte de un conjunto de conceptos que, a partir de 1992, pasaron a determinar las iniciativas de las Naciones Unidas para la paz: 1) Diplomacia preventiva, cuyo objetivo es evitar el surgimiento de conflictos; 2) Promoción de la paz: busca acuerdos pacíficos entre las partes; 3) Manutención de la paz: intenta poner en práctica los acuerdos obtenidos; 4) Imposición de la paz: considera medidas coercitivas y militares para restaurar la paz; 5) Consolidación de la paz: fortalece las estructuras capaces de promover la paz. ${ }^{11}$

Este conjunto de ideas surgió al final de la Guerra Fría, de la cual los Estados Unidos salieron vencedores como potencia mundial. Tal hecho histórico cobró fundamental importancia en la forma de proceder de las Naciones Unidas en lo que toca a la paz, pues, a partir de ese momento, la paz democrática y liberal se convirtió en el paradigma de paz dominante en el ámbito de esta organización. ${ }^{12}$ De este modo, se fortaleció la idea de que las democracias y las economías de mercado serían condiciones esenciales para alcanzar la paz mundial. ${ }^{13}$ Así, pues, con vistas a promover la paz, las Naciones Unidas se dispusieron a actuar en áreas como la organización de elecciones y el fortalecimiento de economías de mercado. ${ }^{14}$

Tras considerar que diversos actores, como países, donantes y organismos internacionales, comenzaron a trabajar

10 "Violence, Peace, and Peace Research", Journal of Peace Research, 6 (1969), pp. 169 y 183; A/47/277, doc. cit., \$§ 55-59.

11 Ibidem, pp. 5 y 15.

${ }^{12}$ R. Blanco, "Del mantenimiento de la paz al proceso de formación del Estado: un esbozo de los esfuerzos de la onU para la paz internacional", Foro Internacional, 54 (2014), p. 274.

13 E. Newman et al., "Introduction", en New Perspectives on Liberal Peacebuilding, Tokio-Nueva York-París, United Nations University Press, 2010, pp. 5-7.

${ }^{14}$ Ibid., pp. 8-9. 
en tales actividades, ${ }^{15}$ las Naciones Unidas buscaron coordinar esos esfuerzos. ${ }^{16}$ En este ámbito de colaboración se enmarca la creación, en 2005, de la Comisión para la Consolidación de la Paz (PBC), junto con el Fondo para la Consolidación de la Paz y la Oficina de Apoyo. ${ }^{17}$ La PBC tiene tres objetivos principales: proponer estrategias para la consolidación de la paz, apoyar en la recuperación luego de los conflictos y coordinar actores en esos procesos. ${ }^{18}$ Asimismo, la PBC se compone de 31 Estados y procede como órgano subsidiario de asesoramiento del Consejo de Seguridad y de la Asamblea General. ${ }^{19}$ Así, pues, la PBC tiene tres formatos: Comité organizador -incluye la totalidad de sus miembros y decide el programa de trabajo de la $\mathrm{PBC}-; 20$ Ajustes específicos -son reuniones de la PBC cuyo propósito es deliberar sobre la situación individual de los países de su agenda-; y Grupo sobre las lecciones aprendidas -busca ampliar el diálogo en asuntos de relevancia para los casos debatidos por la PBC. ${ }^{21}$

Para 2017, seis países figuran en la agenda de la PBC (Burundi, Sierra Leona, Guinea-Bisáu, República Centroafricana, Guinea Ecuatorial y Liberia). Un país puede incluirse en la agenda de la PBC mediante la solicitud de los siguientes órganos: el Consejo de Seguridad; el Comité Económico y

15 R. PARIs, "Understanding the «Coordination Problem» in Postwar Statebuilding”, en R. Paris y T. Sisk (eds.), The Dilemmas of Statebuilding: Confronting the Contradictions of Postwar Peace Operations, Nueva York, Routledge, 2009, p. 57.

16 A/59/565, “A More Secure World: Our Shared Responsibility”, 2004, § 261, en https://www.un.org/ruleoflaw/blog/document/the-secretary-generals-high-level-panel-report-on-threats-challenges-and-change-amore-secure-world-our-shared-responsibility/, consultado el 21.VI.2018.

${ }^{17}$ A/RES /60/180, "Resolução da Assembleia Geral”, 2005, en http:/ / www.securitycouncilreport.org/atf/cf/\% 7B65BFCF9B-6D27-4E9C-8CD3CF6E4FF96FF9\% 7D/PBC\%20ARES60180.pdf, consultado el 15.X.2013.

18 Ibid., $\$ 2$.

${ }^{19} \mathrm{Id}$.

${ }^{20}$ R. Jenkins, Peacebuilding. From Concept to Commission, Nueva York, Routledge, 2013, p. 46.

${ }^{21}$ Ibid. pp. 47-48. 
Social (Ecosoc) o la Asamblea General -con el consentimiento del Estado miembro en cuestión-; los Estados miembros -cuando el Estado esté al borde del conflicto y no se encuentre en la agenda del Consejo de Seguridad-; y el Secretario general. ${ }^{22}$ El Fondo, a su vez, ofrece apoyo financiero a los miembros del sistema de las Naciones Unidas para la consolidación de la paz. ${ }^{23}$ Por último, la Oficina de Apoyo tiene el propósito de aconsejar estratégicamente a la PBC, de administrar el Fondo y de servir al Secretario general en la coordinación de las agencias de la ONU en sus esfuerzos para establecer la paz. ${ }^{24}$

La fundación de ese nuevo marco institucional fue el resultado de un complejo proceso de negociaciones en que se hicieron presentes visiones estatales divergentes acerca de la forma de composición y de funcionamiento de la PBC. A este propósito, Gilda Motta Santos Neves destaca, por lo menos, tres puntos de controversia: la naturaleza preventiva de la PBC ("early warning"); el locus institucional de la PBC en el ámbito jerárquico de las Naciones Unidas; y la composición de la Comisión. ${ }^{25}$

La idea de la función preventiva estuvo presente en los debates iniciales sobre la creación de la PBC y tendría por objetivo “...identificar a los países que se encuentran bajo presión y en riesgo de precipitarse hacia el colapso del Estado". ${ }^{26}$ Aunque países como Brasil y Sudáfrica defendían esa función, predominó la visión que la interpretaba como forma de supervisar la situación interna de los países. Por lo

${ }^{22} \mathrm{~A} / \mathrm{RES} / 60 / 180$, doc. cit., $\S 12$.

23 Pвso, "Peacebuilding: An Orientation", 2010, p. 37, en http:// www.un.org/en/peacebuilding/pbso/pdf/peacebuilding_orientation. pdf, consultado el 7.VIII.2015.

${ }^{24} \mathrm{~A} / \mathrm{RES} / 60 / 180$, doc. cit., $\$ 23$.

${ }^{25}$ Comissão das Nações Unidas para Consolidação da Paz: perspectiva brasileira, Brasilia, FUNAG, 2009, pp. 113 y 123.

${ }^{26}$ Traducción del original en inglés: "...identify countries which are under stress and risk sliding towards State collapse”. Véase A/59/565, doc. cit., $\S 264$. 
tanto, la función señalada fue excluida de las prerrogativas de la PBC. $^{27}$

En lo que respecta al locus institucional de la PBC, Neves explica que "...cabe determinar si la Comisión debería supeditarse (a) únicamente al Consejo de Seguridad; (b) al Ecosoc [Consejo de Seguridad y Social de las Naciones Unidas]; (c) a la Asamblea General; (d) a los tres; o (e) sólo al Consejo de Seguridad y al Ecosoc y, en ese caso, en paralelo o en secuencia”. ${ }^{28}$ La autora destaca, en ese sentido, la oposición entre los miembros permanentes del Consejo de Seguridad, ${ }^{29}$ al cual, según éstos, la PBC tendría que subordinarse de manera exclusiva, y los países en desarrollo, para los cuales la PBC debería estar vinculada a los organismos más representativos de las Naciones Unidas, como el Ecosoc y la Asamblea General. ${ }^{30}$

Respecto de la composición institucional de la PBC, se verificaron también intensas disputas políticas. Se dieron, por ejemplo: 1) la creación de vacantes para los mayores contribuyentes con el presupuesto de las Naciones Unidas, lo que, según Neves, ${ }^{31}$ benefició a los países ricos de esa organización; 2) divergencias entre los miembros permanentes del Consejo de Seguridad, que defendieron un organismo de hasta veinte miembros, y países en desarrollo, que demandaron mayor número de participantes en la PBC; 3) disputas en cuanto a los participantes del Consejo de Seguridad en la PBC, pues, en principio, la composición de ésta incluía cinco miembros de ese organismo, tanto permanentes como eventuales; sin embargo, con el avance de las discusiones, tal estructura se modificó y los cinco miembros permanentes del Consejo pasaron a constituir la $\mathrm{PBC}$, lo que tuvo por resultado la reducción del

27 Neves, op. cit., p. 113.

28 Ibid., p. 127.

${ }^{29}$ Los miembros permanentes del Consejo de Seguridad son China, Estados Unidos, Francia, Reino Unido y Rusia.

${ }^{30}$ Neves, op. cit., p. 123.

31 Ibid., p. 129. 
espacio de participación para los miembros eventuales. ${ }^{32} \mathrm{Se}$ comprueba, por tanto, que la fundación de la PBC evidenció no sólo intereses opuestos entre los diferentes miembros de las Naciones Unidas, sino también el predominio de las posiciones de los Estados económica y políticamente más poderosos en el entorno de esa organización. ${ }^{33}$

Además, la PBC opera con base en la lógica de la paz liberal, lo que condiciona el alcance de su visión. Según Oliver Richmond y Jason Franks, ${ }^{34}$ Roland Paris ${ }^{35}$ y Blanco, ${ }^{36}$ el paradigma de la paz liberal asocia la promoción de la paz con el liberalismo económico, político y social, entendido éste como condición esencial para el establecimiento de una paz duradera. ${ }^{37}$ Blanco asegura que, según esta forma de pensar,

32 Ibid. p. 132.

33 Ibid. p. 117.

34 Liberal Peace Transitions: Between Statebuilding and Peacebuilding, Edinburgo, Edinburgh University Press, 2009, p. 4.

35 At War's End: Building Peace After Civil Conflict, Cambridge, University Press, 2004.

${ }^{36}$ Art. cit., p. 280, y "The Brazilian Engagement with Peace Operations: A Critical Analysis”, Revista Brasileira de Política Internacional, 60 (2017), núm. 2, p. 8.

${ }^{37}$ Para profundizar en lo que es el paradigma de paz liberal, se recomiendan los estudios de M. Duffield, Global Governance and the New Wars, Londres, Zed Books, 2001; J. Heathershaw, "Unpacking the Liberal Peace: The Dividing and Merging of Peacebuilding Discourses", Millennium. Journal of International Studies, 36 (2008), pp. 597-621 (2008); PARIS, op. cit.; O. Richmond, "The Problem of Peace: Understanding the «Liberal Peace»", Conflict, Security E Development, 6 (2006), pp. 291-314, y The Transformation of Peace, Nueva York, Palgrave Macmillan, 2007; A. T. GoMEs, "Da paz liberal à virada local: avaliando a literatura crítica sobre peacebuilding", Monções: Revista de Relações Internacionais da ufgd, 2 (2013), núm. 4, pp. 46-76; Blanco, "Del mantenimiento de la paz al proceso de formación del Estado..."; y Kemer et al., art. cit. En lo que se refiere a las críticas a la paz liberal, se sugieren, además, S. Chesterman, You, The People: The United Nations, Transitional Administration, and State-Building, Oxford, University Press, 2004, y "Ownership in Theory and in Practice: Transfer of Authority in un Statebuilding", Journal of Intervention and Statebuilding, 1 (2007), pp. 3-26; D. CHANDLER, "A Crítica não-crítica da «Paz Liberal»", Universitas: Relações Internacionais, 11 (2013), núm. 2, pp. 39-51 
"los Estados liberales son más desarrollados, pacíficos y -sobre todo- estables, tanto en el ámbito nacional como en el internacional". ${ }^{38}$ Para el estudioso, el paradigma de la paz liberal se fundamenta en dos pilares, los cuales vinculan la paz a la existencia de Estados democráticos y de relaciones económicas entre los países. ${ }^{39} \mathrm{El}$ primer pilar se establece a partir de los trabajos de Immanuel Kant ${ }^{40}$ y se fundamenta en la teoría de la paz democrática, según la cual las democracias son menos proclives a hacer guerra. ${ }^{41} \mathrm{El}$ segundo, a su vez, tiene por fundamento trabajos como los del Barón de Montesquieu ${ }^{42}$ o de Joseph Schumpeter, ${ }^{43}$ y dispone, básicamente, que Estados interrelacionados por su economía tienen mayor propensión a la paz, habida cuenta de, por ejemplo, los daños comerciales que pueden derivar de los conflictos internacionales. ${ }^{44}$

(2013); PAris, op. cit.; M. Pugh, "Peacekeeping and Critical Theory", en A. Bellamy y P. Williams (eds.), Peace Operations and Global Order, Nueva York, Routledge, 2005, y "The Political Economy of Peacebuilding: A Critical Theory Perspective”, International Journal of Peace Studies, 10 (2005), núm. 2, pp. 23-42; I. TAYLOR, "What Fit for the Liberal Peace in Africa?", Global Society, 21 (2007), pp. 553-566, y "Liberal Peace, Liberal Imperialism: A Gramscian Critique", en O. Richmond (ed.), Palgrave Advances in Peacebuilding: Critical Developments and Approaches, Londres, Palgrave Macmillan, 2010; O. Richmond, Peace in Advances in Peacebuilding: Critical Developments and Approaches, Londres, Palgrave Macmillan, 2010; y R. MAC GINTy, International Peacebuilding and Local Resistance: Hybrid Forms of Peace, Nueva York, Palgrave, 2011.

38 "Del mantenimiento de la paz al proceso de formación del Estado...", p. 280.

39 Ibid. p. 281.

40 Perpetual Peace: A Philosophical Essay, Londres, George Allen \& Unwin Ltd., 1905 [1795].

41 Blanco, "Del mantenimiento de la paz al proceso de formación del Estado...”, p. 281, y Kemer et al., art. cit., p. 140.

42 The Spirit of the Laws, Cambridge, University Press, 2002 [1748].

43 "The Sociology of Imperialism", en J. Schumpeter (comp.), Imperialism and Social Classes: Two Essays by Joseph Schumpeter, Cleaveland, Meridian Books, 1966 [1919].

44 Blanco, "Del mantenimiento de la paz al proceso de formación del Estado...”, p. 282, y Kemer et al., art. cit., p. 140. 
Con base en estas premisas, iniciativas liberales -como la búsqueda de la democratización de los Estados, la promoción de economías de mercado y la defensa de los derechos humanos- predominan en el ámbito de organizaciones internacionales como las Naciones Unidas. ${ }^{45}$ De hecho, en las Naciones Unidas se concibe la paz según el concepto de Oliver Richmond ${ }^{46}$ de consenso de la consolidación de la paz, que se fundamenta en la existencia de una base cultural y normativa universalmente aceptada para la paz liberal. Tal consenso implica la búsqueda de modelos de gobiernos liberales, generalmente reputados, que Blanco denomina "modelo de tamaño único"; ${ }^{47}$ Cavalcante, "convicción política", ${ }^{48}$ y Oliver Ramsbotham, "procedimiento operativo estándar". ${ }^{49}$ La consabida hipótesis según la cual los gobiernos liberales hacen viable de manera automática la consolidación de la paz en países involucrados en conflictos violentos acarrea a estos últimos diversos problemas. Roland Paris señala los problemas que emergen de la adopción de esos "modelos de tamaño único", ${ }^{50}$ entre los cuales se encuentran el limitado conocimiento sobre la realidad local y la escasa participación de sus actores sociales en procesos decisivos. Estas críticas son esenciales si se quiere comprender los límites de la actuación de la $\mathrm{PBC}$, la cual, a pesar de los propósitos con que fue fundada, es incapaz de dar respuesta a los señalamientos, como apunta

45 Newman et al., art. cit., pp. 8-9.

46 The Transformation of Peace, p. 112.

47 "The Un Peacebuilding Process: An Analysis of its Shortcomings in Timor-Leste”, Revista Brasileira de Política Internacional, 58 (2015), núm. 1, p. 45 .

48 Coming into Life: The Concept of Peace Building in the United Nations, from "An Agenda for Peace" to the Peace Building Commission, tesis, Coimbra, Faculdade de Economia da Universidade, 2013, p. 220.

49 "Reflections on un post-settlement peacebuilding", International Peacekeeping, 7 (2000), p. 170. Véase también Blanco, "The un Peacebuilding Process...”, p. 45.

50 "Saving Liberal Peacebuilding”, Review of International Studies, 36 (2010), pp. 337-365. Véase, además, Blanco, "The un Peacebuilding Process...", p. 45 . 
Blanco. ${ }^{51}$ De hecho, según el autor, aunque la PBC se concibió para promover la consolidación de la paz, sus esfuerzos continuaron, en parte, dentro del mismo marco normativo de la paz liberal. ${ }^{52}$ Fernando Cavalcante demostró ese argumento por medio del análisis de los documentos estratégicos para la consolidación de la paz elaborados por la PBC para los seis países de su agenda. Todos los documentos presentaron prioridades asociadas con la paz democrática y liberal, como la celebración de elecciones y la realización de reformas administrativas. ${ }^{53}$ En consecuencia, el autor afirmó que las estrategias elaboradas por la PBC reflejan la paz democrática y liberal como el paradigma de la consolidación de la paz en las Naciones Unidas. ${ }^{54}$ Éste fue el caso de Guinea-Bisáu, en que la adopción de la universalidad de la paz liberal por parte de la PBC llevó a la Comisión a distanciarse de la realidad local, con lo cual se corroboraron las críticas de Paris y de Blanco. 55

\section{EL ENTORNO POLÍTICO DE DESARROLLO Y DE SEGURIDAD DE GUINEA-BisÁU}

Guinea-Bisáu se ubica en el África subsahariana, posee cerca de 1.8 millones de habitantes ${ }^{56} \mathrm{y}$, como idioma oficial, el portugués. ${ }^{57}$ Para alcanzar un mayor conocimiento de este país,

51 "Del mantenimiento de la paz al proceso de formación del Estado...", p. 289.

${ }^{52} I d$.

53 Op. cit., p. 251.

54 Ibid., pp. 247-248.

55 Véanse, respectivamente, PARIs, "Saving Liberal Peacebuilding", y Blanco, "Del mantenimiento de la paz al proceso de formación del Estado...".

${ }^{56}$ Banco Mundial, "World Development Indicators", 2015, en http:/ / wdi.worldbank.org/tables, consultado el 24.XI.2015.

${ }^{57}$ Guinea-Bisáu forma parte tanto de la cPlP como de los Países Africanos de Lengua Oficial Portuguesa (PALOP), que también incluye a Mozambique, Angola, Cabo Verde, Santo Tomé y Príncipe y Guinea Ecuatorial. 
es preciso considerar sus esferas política, de desarrollo y de seguridad; es decir precisamente lo que constituye el núcleo del presente apartado, el cual dispondrá las bases para el análisis que emprenderemos en la sección subsiguiente acerca de las prácticas de la PBC.

En el ámbito político, es importante destacar la lucha anticolonial de Guinea-Bisáu, que la llevó a emprender los movimientos de independencia para emanciparse de Portugal y a erigir el Estado de Guinea. Dominada desde el siglo xvi, Guinea-Bisáu inició su independencia de Portugal en la segunda mitad del siglo xx, mediante la formación, en 1956, del Partido Africano para la Independencia de Guinea-Bisáu y Cabo Verde (PAigC). ${ }^{58}$ El Paigc, que buscaba la independencia de estas dos antiguas colonias portuguesas, tuvo como primer líder a Amílcar Cabral, ampliamente reconocido por su importancia histórica en el proceso de descolonización de África. ${ }^{59}$ Según Pereira y Vittoria, ${ }^{60}$ al defender la unidad de los miembros del PAIGC en pro de la independencia, a pesar de sus diferencias étnicas y religiosas, Cabral se convirtió en figura esencial para que Guinea-Bisáu lograra el éxito en su proceso de emancipación. Además, al apoyar, por ejemplo, la movilización de otros pueblos africanos, como el Movimiento Popular de Liberación de Angola (MPLA), Cabral también contribuyó en modo más amplio al avance de la agenda de

Véase MRe, "Comunidade dos Países de Língua Portuguesa", 2016, en http:/ / www.itamaraty.gov.br/pt-BR/politica-externa/mecanismos-interregionais /3676-comunidade-dos-paises-de-lingua-portuguesa-cplp, consultado el 17.VI.2016.

58 PAIGC, "História do PAIGC", 2018, en http://www.paigc.gw/historia.html, consultado el 21.VI.2018.

${ }^{59}$ A. A. Pereira y P. Vittoria, A luta pela descolonização e as experiências de alfabetização na Guiné-Bissau: Amilcar Cabral e Paulo Freire, 2012, Estud. hist. (Rio J.), 25 (2012), núm. 50, p. 294, y J. Neves, "Ideologia, ciência e povo em Amílcar Cabral”, História, Ciências, Saúde - Manguinhos, 24 (2017), p. 334.

${ }^{60}$ Art. cit., p. 294. 
descolonización africana. ${ }^{61}$ Por lo tanto, aunque los movimientos anticoloniales guineanos se desenvolvieron en un ámbito de debilitamiento del gobierno portugués, ${ }^{62}$ la actuación de Cabral fue decisiva para que Guinea-Bisáu fuera la primera colonia portuguesa africana en declarar su independencia. ${ }^{63}$ Con la emancipación de Guinea-Bisáu en 1973 y el asesinato de Amílcar Cabral el mismo año, Luis Cabral, hermano de aquél, se convirtió en el primer presidente del país, el cual pasó a tener un régimen de partido único, el PAIGG. ${ }^{64}$

Guinea-Bisáu ha padecido crisis políticas desde su declaración de independencia en 1973, y la participación de los militares desempeña un papel primordial en ese respecto. ${ }^{65}$ Desde 1973, ningún presidente electo ha completado el período correspondiente a su mandato, pues prácticamente todos han sido depuestos por los militares. ${ }^{66}$ El primer golpe militar se remonta a 1980, cuando el brigadier João Bernardo Vieira, "Nino" Vieira, destituyó al presidente Luis Cabral. ${ }^{67}$ Después del golpe, Nino Vieira permaneció en el poder de Guinea-Bisáu de 1980 a 1999 y, aún, de 2005 a 2009. A lo largo

${ }^{61} I d$. Véase también PAigc, doc. cit.

62 En 1974, dio comienzo, en Portugal, la Revolución de los Claveles, que representó la insatisfacción del pueblo portugués para con el gobierno dictatorial de Marcello Gaettano, quien ejerció el poder de 1968 a 1973 y, además, había sido el sucesor del gobierno, también dictatorial, de Antonio de Oliveira Salazar (1932-1968). Véase L. Secco, "Trinta anos da Revolução dos Cravos", Revista ADUSP, 2004, núm. 33, pp. 6-12, en http:// www.adusp.org.br/files/revistas/33/r33a01.pdf, consultado el 18.VI.2018.

${ }^{63}$ Pereira y Vittoria, art. cit., p. 294.

${ }^{64}$ P. M. Ferreira, "Guinea-Bissau: Between Conflict and Democracy”, African Security Review, 13 (2004), p. 45.

${ }^{65}$ D. O'Reagan y P. Thompson, "Advancing Stability and Reconciliation in Guinea-Bissau: Lessons from Africa's First Narco-State", Washington, D. C., Africa Center for Strategic Studies, informe especial núm. 2, 2013, p. 6, en http://www.globalinitiative.net/download/drugs/subsaharan-africa/ACSS $\% 20 \% 20$ Advancing $\% 20$ Stability $\% 20$ and $\% 20$ Reconciliation\%20in\%20Guinea\%20Bissau\%202013.pdf, consultado el 10.VIII.2015.

${ }^{66}$ Ibid., p. 3.

${ }^{67}$ Ibid., p. 9. 
de ese primer período, el país adoptó un régimen multipartidista, en 1994, y un régimen semipresidencialista, en 1996, año en que se publicó la Constitución de Guinea-Bisáu. ${ }^{68}$

Además de lo anterior, otro episodio que significó un hito en la historia política del país ocurrió en 1998, en el ámbito de una guerra civil nacional. Esta crisis se inició cuando el presidente de Senegal, Abdou Diouf, presionó a Nino Vieira para que detuviera el tráfico de armas entre los miembros de las Fuerzas Armadas de Guinea-Bisáu y Senegal. ${ }^{69}$ En respuesta, Nino Vieira responsabilizó del trasiego al Jefe de las Fuerzas Armadas de Guinea-Bisáu, Ansumane Mané, lo que montó un escenario propicio para el conflicto, que duraría nueve meses y dejaría más de 2000 muertos, entre las fuerzas que eran leales al presidente Nino y las que lo eran a los militares. ${ }^{70}$

Según Cavalcante, la participación de instituciones multilaterales, tanto de países africanos como de países lusófonos, fue de relevancia para lograr la pacificación de esta guerra civil. ${ }^{71}$ De este modo, la Comunidad Económica de los Estados de África Occidental (CEDEAO) y la Comunidad de Países de Lengua Portuguesa (CPLP) hicieron posible la mediación del Acuerdo de paz de Abuja, en Nigeria, en agosto de 1998. El acuerdo estableció una división de poder entre las fuerzas beligerantes, la creación de un gobierno de unidad nacional, la retirada de las fuerzas extranjeras de Guinea-Bisáu y el establecimiento de una fuerza de peacekeeping

${ }^{68}$ República da Guiné-Bissau, "Constituição da República da GuinéBissau”, 1996, en http://www.anpguinebissau.org/leis/constituicao/constituicaoguine.pdf/view, consultado el 24.XI.2015.

${ }^{69}$ G. O. YABI, The Role of ECowAS in Managing Political Crisis and Conflict: The Cases of Guinea and Guinea-Bissau, trad. de Roger Addah, Abuja, Nigergia, Friedrich-Ebert-Stiftung, 2010, en http://library.fes.de/pdffiles/bueros/nigeria/07448.pdf, consultado el 11.VIII.2015; FERreira, art. cit., p. 46.

${ }^{70} I d$. Véase, asimismo, FMI, "Guiné-Bissau. Temas selecionados", informe núm. 15/195, 2015, pp. 10-11, en https://www.imf.org/external/lang/portuguese/pubs/ft/SCR/2015/cr15195p.pdf, consultado el 26.XI.2015.

${ }^{71}$ Art. cit., p. 28. 
de la CEDEAO en Guinea-Bisáu para la implementación del acuerdo. ${ }^{72}$

En lo que respecta a las Naciones Unidas, en abril de 1999, el Consejo de Seguridad autorizó la fundación de la Oficina de Apoyo para la Consolidación de la Paz en GuineaBisáu (UnOGBIs) ${ }^{73}$ Esta oficina estaría bajo la dirección de un representante del Secretario general y tendría los siguientes propósitos: 1) apoyar la aplicación del Acuerdo de Abuja y 2) promover, del mejor modo posible, la integración de las actividades de las Naciones Unidas durante el período de transición y hasta la celebración de los comicios en GuineaBisáu. ${ }^{74}$ Esta iniciativa constituyó un marco particularmente relevante para el análisis llevado a cabo en este artículo en la medida en que implicó el comienzo de la intervención de las Naciones Unidas en el país con el objetivo de tratar específicamente el tema de la consolidación de la paz.

72 Ferreira, art. cit., p. 46; Cavalcante, art. cit., p. 28; s/1998/1028, "Letter dated 3 November 1998 from the Permanent Representative of Nigeria to the United Nations Addressed to the President of the Security Council", 1998, en http://www.un.org/en/ga/search/view_doc.asp?symbol= S/1998/1028, consultado el 23.VI.2018.

73 S/RES/1233, "Resolução do Conselho de Segurança”, 1999, en http:/ / daccess-dds-ny.un.org/doc/UNDOC/GEN/N99/096/57/PDF/ N9909657.pdf?OpenElement, consultado el 23.VI.2018; s/1999/232, "Carta do Secretário-Geral ao Presidente do Conselho de Segurança”, 1999, en http:/ / www.securitycouncilreport.org/atf/cf/\% 7B65BFCF9B-6D27-4E9C8CD3- CF6E4FF96FF9\%7D/GB\%20S1999\%20232.pdf, consultado el 12. VIII.2015.

${ }^{74}$ S/RES/1233, doc. cit., $\$ 7$. De forma ampliada, el mandato de UNOGBIS comprendía: 1) ayudar en la elaboración de un ambiente propicio para la restauración y consolidación de la paz, de la democracia del Estado de Derecho y en la organización de elecciones libres y transparentes; 2) trabajar con el gobierno y los socios nacionales para poner en práctica el Acuerdo de Abuja; 3) buscar el compromiso del gobierno y de otras partes involucradas para adoptar un programa de recolección voluntaria y destrucción de armas; 4) buscar la integración de las actividades de las Naciones Unidas en el país, en particular durante el período de transición (s/1999/232, doc. cit., p. 1). 
Así, con la conclusión de la guerra civil, las elecciones presidenciales de noviembre de 1999 dieron como ganador a Kumba Yalá, quien ofreció puestos políticos a personas de la etnia Balanta, ${ }^{75}$ con que fomentó la rivalidad entre las etnias del país. ${ }^{76}$ Este episodio de crisis política, verdadero caldo de cultivo, gestó las condiciones para un nuevo golpe de Estado, en 2003, con el cual el general Veríssimo Correia Seabra depondría al presidente Kumba Yalá. ${ }^{77}$ En este ambiente de pugna, fueron nuevamente la CPLP y la CEDEAO las que desempeñaron un papel fundamental para la mediación del conflicto, toda vez que propiciaron las negociaciones que harían posible la formación de un Gobierno de Transición en Guinea-Bisáu, en que Francisco Fadul ocupó el cargo de primer ministro. ${ }^{78}$ Después del golpe, se convocó a nuevas elecciones presidenciales en 2005, que se distinguieron por reinstalar a Nino Vieira en el poder, electo como candidato independiente. ${ }^{79}$ Sin embargo, en 2009, el Jefe de las Fuerzas Armadas, Tagmé na Wai, y el presidente Vieira fueron asesinados. ${ }^{80}$ Tras este acontecimiento, en 2009 se convocó inmediatamente a nuevas elecciones, las cuales proclamaron la

${ }^{75}$ La etnia Balanta es uno de los cerca de cuarenta grupos étnicos que conforman el país y representa, aproximadamente, el $30 \%$ de la población.

${ }^{76}$ Ferreira, art. cit., p. 55; Crisis Group, "Guinea-Bissau: In Need of a State", informe sobre Africa núm. 142, 2 de julio de 2008, p. 15, en http://www.crisisgroup.org/ /media/Files/africa/west-africa/guineabissau/Guinea-Bissau\%20In\%20Need\%20of\%20a\%20State.pdf, consultado el 11.VIII.2015.

${ }^{77} \mathrm{Id}$.

${ }^{78} \mathrm{Id}$.

${ }^{79}$ Ibid., p. 16.

${ }^{80}$ Ibid., p. 8. Según Crisis Group ("La réforme du secteur de la sécurité en Guinée-Bissau: une occasion à saisir", informe sobre África núm. 109, 2015, p. 17, en http://www.crisisgroup.org/fr/regions/afrique/ afrique-de-louest/guinee-bissau/b109-security-sector-reform-in-guineabissau-an-opportunity-not-to-be-missed.aspx, consultado el 26.XI.2015), Tagmé na Wai fue asesinado en un bombardeo y, horas después, sus tropas entraron en la residencia presidencial para torturar y matar al presidente Nino Vieira. 
victoria de Malam Bacai Sanhá, uno de los héroes de la liberación nacional, y del primer ministro Carlos Gomes Júnior. ${ }^{81}$

Esta crisis política continuó en 2010, cuando el lugarteniente de las Fuerzas Armadas, Antonio Indjai, ordenó a sus soldados la detención, entre otros, del Jefe de las Fuerzas Armadas, Zamora Induta, y del primer ministro, Carlos Gomes Júnior. ${ }^{82}$ Además de permanecer impune, Indjai fue designado el nuevo Jefe de las Fuerzas Armadas por el presidente Malam Bacai Sanhá, lo que complicó aún más la crisis política nacional. ${ }^{83}$ En este entorno, Angola, que ocupaba la presidencia de la CPLP, firmó un acuerdo de cooperación en Reforma del Sector de Seguridad (Rss) con Guinea-Bisáu, ${ }^{84}$ el cual permitía el desplazamiento a ésta de 200 militares para cumplir con la MISSANG ${ }^{85}$ misión que tenía como propósito realizar entrenamientos y rehabilitar infraestructuras militares, tales como cuarteles. ${ }^{86}$

En la estela de ese proceso, en diciembre de 2011, hubo un intento malogrado de golpe militar contra el presidente Sanhá, ${ }^{87}$ quien, sin embargo, no pudo terminar su mandato a causa de cierta enfermedad que lo llevó a sucumbir en ene-

${ }^{81}$ O'Reagan y Thompson, informe citado, p. 8.

${ }^{82}$ Crisis Group, "Para além dos compromissos: as perspectivas de reforma na Guiné-Bissau”, informe sobre África núm. 183, 2012, p. 1, en http://www.crisisgroup.org/ /media/Files/africa/west-africa/guineabissau/Portuguese \% 20translations/183-au-dela-des-compromis-les-perspectives-de-reforme-en-guinee-bissau-portuguese, consultado el 30.XI.20 15; OPLOP, "Instabilidade e insegurança: a Guiné-Bissau entre o abril de 2010 e de 2012", 2013, en http://www.oplop.uff.br/boletim/2160/instabilidade-inseguranca-guine-bissau-entre-abril-de-2010-de-2012, consultado el 26.XI.2015.

${ }^{83} \mathrm{Id}$.

${ }^{84}$ OpLop, "Angola oficializa apoio militar para Rss na Guiné-Bissau", 2011, en http://www.oplop.uff.br/boletim/115/angola-oficializa-apoiomilitar-para-reforma-no-setor-de-seguranca-defesa-em-guine-bissau, consultado el 21.XII.2015.

${ }^{85}$ MISSANG es el acrónimo en portugués para la Misión de Angola en Guinea-Bisáu.

${ }^{86}$ Oplop, art. cit.

${ }^{87}$ Crisis Group, "Para além dos compromissos...", p. 1. 
ro de 2012. Vacante el puesto, se iniciaron nuevas disputas electorales por la presidencia que culminaron, una vez más, en otro golpe militar en abril de 2012, cuando las tropas del general Indjai arrestaron al candidato y ex primer ministro Carlos Gomes Júnior, quien había obtenido la mayoría de los votos en la primera vuelta. Después de este acontecimiento, un comando militar tomó el control del gobierno y anunció la suspensión de la Constitución. ${ }^{88}$ Este golpe de Estado fue ampliamente repudiado en la comunidad internacional, por lo cual las actividades de Guinea-Bisáu en la Unión Africana y en la CEDEAO se interrumpieron, ${ }^{89}$ además de que el Consejo de Seguridad de las Naciones Unidas, por medio de la Resolución 2048, ${ }^{90}$ impuso sanciones a los perpetradores del golpe, después del cual la CEDEAo envió la "Misión de Seguridad para Guinea-Bisáu", es decir, la ЕСОмгв, ${ }^{91}$ fuerza militar compuesta de 629 soldados procedentes de Costa de Marfil, Nigeria, Senegal y Togo, con el objetivo de sustituir a los militares de Angola (MISSANG), de apoyar la restauración del orden constitucional y de permitir el desplazamiento por el país de agencias humanitarias. ${ }^{92}$ En el ámbito de los países lusófonos, la CPLP también condenó los actos subversivos que se estaban dando en GuineaBisáu y se posicionó como el coordinador diplomático de los esfuerzos internacionales para la resolución de la crisis

88 O'Reagan y Thompson, informe citado, p. 16.

${ }^{89}$ Unión Africana, "Report of the Chairperson of the Commission on the Situation in Guinea-Bissau", 2014, § 3, en http://www.peaceau.org/ uploads/auc-report-guinea-bissau-psc-17-06-2014.pdf, consultado el 23. VI.2018.

${ }^{90}$ S/RES/2048, "Resolução do Conselho de Segurança", 2012, en http://www.un.org/ga/search/view_doc.asp?symbol=S/RES/2048 (2012), consultado el 23.VI.2018.

${ }^{91}$ Есомів es el acrónimo en inglés para Ecowas Security Mission in Guinea Bisáu.

92 Cedeao, "Regional Defence Chiefs to Meet on ecowas Mission in Guinea Bissau", 2013, en http://news.ecowas.int/presseshow.php?nb=144 \&lang=en\&annee=2013, consultado el 22.VI.2018. 
en el país. ${ }^{93}$ A pesar de estos esfuerzos multilaterales, el grave cuadro político persistió en Guinea-Bisáu hasta realizarse nuevas elecciones, que sólo ocurrieron en 2014. Así, pues, la política guineana estuvo condicionada por la interferencia de las Fuerzas Armadas en el gobierno, lo que contribuyó a generar inestabilidad continua en el país. ${ }^{94}$

Además de la crisis política propiciada por el golpe militar, Guinea-Bisáu se destaca como una de las principales rutas mundiales (de paso) para el tráfico ilícito internacional de estupefacientes. ${ }^{95} \mathrm{El}$ comercio de cocaína es ejemplo emblemático de ello, pues ha ido acrecentándose desde mediados de la década del 2000. De hecho, la Oficina de las Naciones Unidas contra la Droga y el Delito (UNODC) estima que, entre 2005 y 2011, entraron al país 14 toneladas de cocaína, ${ }^{96}$ y O'Reagan y Thompson ${ }^{97}$ señalan que, por lo menos, han ingresado 25 toneladas de cocaína al país entre abril y julio de 2012. Esta situación se agravó por la injerencia de las autoridades de Guinea-Bisáu en el tráfico ilícito. Como caso elocuente de esta intromisión puede citarse el de la aprehensión por parte de los Estados Unidos, en 2013, del ex Comandante de Marina de Guinea-Bisáu, almirante José Américo Bubo Na Tchuto, y del Jefe de las Fuerzas Armadas, general Antonio Indjai, debido a su participación en el tráfico de armas y de cocaína. ${ }^{98}$

${ }^{93}$ Mre, "Resolução da CPLP sobre a Situação na Guiné-Bissau", 2012, en http://www.itamaraty.gov.br/pt-BR/notas-a-imprensa/3023-resolucao-da-cplp-sobre-a-situacao-na-guine-bissau, consultado el 21.VI.2018.

${ }^{94}$ K. Rizzi, "A instabilidade contínua na Guiné-Bissau", Meridiano 47, 11 (2010), núm. 117, p. 23; O'Reagan y Thompson, informe citado, p. 6.

${ }^{95}$ M. SHaw, "Drug Trafficking in Guinea-Bissau, 1998-2014: The Evolution of an Elite Protection Network", The Journal of Modern African Studies, 53 (2015), pp. 339-364.

${ }^{96}$ Unode, "Criminalidade Organizada Transnacional na África Ocidental: Avaliação da Ameaça", 2013, p. 10, en https://www.unodc.org/ documents/data-and-analysis/tocta/West_Africa_TOCTA_2013_PT.pdf, consultado el 24.XI.2015.

${ }^{97}$ Informe citado, p. 3.

${ }^{98}$ EuA, "United States of America vs Jose Americo Bubo Na Tchuto, Papis Djeme and Tchamy Yala", 2015, en http://www.justice.gov/sites/de- 
Así, el desarrollo guineano, que constituye la segunda esfera de análisis, debe comprenderse en el ámbito de estas crisis, teniendo en cuenta que los problemas políticos y, además, el tráfico ilícito internacional afectan negativamente tanto la calidad de vida de la población como las instituciones de Guinea-Bisáu. ${ }^{99}$ Con base en este ambiente de descomposición, en 2014 el país presentó uno de los valores más ínfimos para el Índice de Desarrollo Humano del mundo, en el centésimo septuagésimo séptimo lugar de 187 países. ${ }^{100} \mathrm{Si}$ en 2010 el 30\% de los guineanos vivía una situación de pobreza extrema, ${ }^{101}$ en 2013 esta cifra alcanzó cerca del 45\%.102 Además, se menciona, por ejemplo, que el país convive, incluso, con un cuadro de elevada mortandad infantil (123 casos por 1000, contra una media en el África subsahariana de 92 por 1000) y con una engrosada cantidad de casos de sida (0.31 de nuevas infecciones anuales por cada 100 personas, frente a una media regional de 0.29). ${ }^{103}$ Asimismo, sólo el $20.8 \%$ de la población tiene acceso al tratamiento de aguas residuales, lo que está en relación con el hecho de que el $80 \%$ de los guineanos viva en favelas. ${ }^{104}$ Así, pues, estos indicadores evidencian, en la actualidad, la gravedad de los problemas que enfrenta Guinea-Bisáu, tanto socialmente como en lo que hace a la capacidad de funcionamiento del aparato estatal correspondiente a la atención de las necesidades básicas de la población: salud, saneamiento y educación.

fault/files/usao-sdny/legacy/2015/03/25/U.S.\%20v. \%20Na\%20Tchuto $\% 20$ et $\% 20$ al\%20Indictment.pdf, consultado el 24.XI.2015.

99 O'REaGan y Thompson, informe citado.

100 PNUD, art. cit., p. 2.

101 La pobreza extrema comprende a aquellas personas que viven con menos de US\$1 por día. Véase Banco Mundial, art. cit., p. 26.

102 Id.

103 MDG, "Millenium Development Goals: Snapshot for Guinea-Bissau", 2015, en http://mdgs.un.org/unsd/mdg/Resources/Static/Products/Progress2015/Snapshots/GNB.pdf, consultado el 30.XI.2015. ${ }^{104}$ Id. 
Se debe observar, además, que los problemas que padece Guinea-Bisáu alcanzan las diferentes regiones del país y capas de la población de modos distintos. Como ejemplo de ello, se advierte que algunas prácticas que se dan en el país afectan a grupos sociales específicos, como en los casos de la mutilación genital femenina y de los matrimonios forzados en la infancia. ${ }^{105}$ Por tanto, es fundamental eludir el error que representa la "homogeneización de lo local", ${ }^{106}$ pues no puede considerarse que las necesidades sociales de la población guineana sean uniformes.

A pesar de la situación de pobreza en Guinea-Bisáu, la "Reforma del Sector de Seguridad" (Rss) -en el marco de la tercera esfera de análisis de este artículo-, ocupó un espacio prioritario en las iniciativas del gobierno para la promoción de la paz. Según el gobierno guineano, la Rss sería el medio adecuado por el cual pacificar las Fuerzas Armadas y establecer las condiciones políticas para el desarrollo. ${ }^{107}$ Subyace a esta afirmación la idea de que los problemas ${ }^{108}$ de las Fuerzas Armadas serían una de las causas de las crisis nacionales; entonces, resolverlos permitiría una paz duradera.

De esta forma, la Rss fue defendida como método esencial para impedir la continuidad de las inestabilidades y favorecer

${ }^{105}$ LGDH, "Relatório sobre a Situação dos Direitos Humanos na GuinéBissau 2008/2009”, 2008, en http:/ / www.lgdh.org/p/relatorios.html, consultado el 9.I.2016.

106 C. Simons y F. SAnker, "Questioning the Local in Peacebuilding”, 2014, p. 5, en http://www.spp1448.de/fileadmin/media/galleries/SPP_ Administration/Working_Paper_Series/SPP1448_WP10_Simons_Zanker. pdf, consultado el 22.II.2016.

107 República da Guiné-Bissau, "Documento de Estratégia Nacional de Redução da Pobreza", 2005, § 86, en http:/ / www.stat-guinebissau.com/ denarp/denarp.pdf, consultado el 13.VIII.2015.

108 Como ejemplo de algunos de ellos, O’Reagan y Thompson (informe citado, p. 7) apuntaron la falta de actualización y el crecido número de los que integraban las Fuerzas Armadas: en 2008, el $45 \%$ de los militares tenía más de 20 años de profesión, y el número de militares oficiales doblaba el de los no oficiales. 
el desarrollo. ${ }^{109}$ Por ello, en 2005, el gobierno de Guinea-Bisáu elaboró, con el apoyo del Reino Unido, el Documento Estratégico de Reestructuración y de Modernización del Sector de Defensa y de Seguridad, ${ }^{110}$ la "Estrategia de Rss", ${ }^{111}$ la cual previó: 1) el redimensionamiento del sector de seguridad y defensa nacional; 2) la modernización de este sector por medio de reformas e inversiones; 3) las inversiones en el sector de Justicia; ${ }^{112}$ 4) la movilización de recursos internacionales para la Rss; 5) el desarrollo de la sociedad civil en la Rss. ${ }^{113}$

Christoph Kohl ha dicho, sin embargo, que esta etrategia no se concretó. Según el autor, los actores internacionales que apoyaron su implementación no tenían conocimiento suficiente acerca de la realidad local, además de que se subestimó la complejidad de la Rss. ${ }^{114}$ Esta complejidad se relaciona con el hecho de que la Rss es un tema politizado, situado en el centro del debate entre actores políticos nacionales e internacionales con intereses específicos en su implementación. En este sentido, Kohl recuerda que las estructuras políticas y militares estuvieron, históricamente, "amalgamadas". ${ }^{115}$ El expresidente Nino Vieira, por ejemplo, fue uno de los principales comandantes de la guerra de independencia que perpetró

109 República da Guiné-Bissau, "Reestructuring and Modernization...", p. 25.

${ }^{110} \mathrm{Id}$.

${ }^{111}$ Pвso, "Mapping of Resources \& Gaps For Peacebuilding in Guinea-Bissau", 2008, § 7, en http://www.un.org/en/peacebuilding/cscs/gb/ key_docs/mapping_resources_2008.pdf, consultado el 17.I.2016.

${ }^{112}$ Los problemas del sector de Justicia de Guinea-Bisáu incluían la falta de independencia del Poder Judicial, la carencia de recursos financieros y de infraestructura y una legislación obsoleta (República da Guiné-Bissau, "Reestructuring and Modernization...", p. 10).

${ }^{113} \mathrm{Id}$.

114 "The Reform of Guinea-Bissau's Security Sector: Between Demand and Practice", Peace Research Institute Frankfurt, informe núm. 126, 2014, pp. 11 y 13, en http://issat.dcaf.ch/content/download/73329/124 7623 /file/prif126\% 20The\%20Reform \% 20of\% 20GB\%20Security $\% 20$ Sector_Kohl.pdf, consultado el 9.I.2016.

${ }^{115}$ Ibid., p. 4. 
el primer golpe militar en el país, en 1980, y permaneció por más de veinte años en el poder. ${ }^{116}$ Así, la Rss fue definida como prioridad por gobiernos cuyos miembros estuvieron asociados al núcleo de esa reforma.

En el ámbito internacional, Kohl mencionó a actores que vieron en la Rss una oportunidad de promover sus propios intereses. ${ }^{117}$ Según el autor, Angola, por ejemplo, apoyó el proceso de la Rss en Guinea-Bisáu por medio del envío de la Misión Militar Angolana (MIssang) en 2011. De este modo, las motivaciones de Angola serían, por un lado, de naturaleza económica, debido a los recursos naturales guineanos, como el petróleo, y geoestratégica, por otro, para afirmar su poder regional respecto de Senegal o Nigeria. ${ }^{118}$

Esta politización de la Rss también puede observarse en el debate sobre su "apropiación local". Aunque controvertida, la noción de "apropiación local” está asociada a la idea de que la sociedad local debe ser responsable de la conducción de sus procesos, en este caso, la Rss. ${ }^{119}$ Sin embargo, Mobekk cuestiona quién es el agente de la apropiación local de la Rss recordando que ese concepto remite a las relaciones tanto entre actores "locales" y "externos" de un país, cuanto entre las capas de la sociedad local. ${ }^{120}$ Así, es necesa-

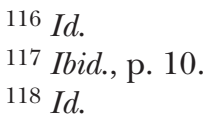

119 S. von Billerbeck, "Whose Peace? Local Ownership and UN Peacebuilding”, investigación presentada en la conferencia The Future of Statebuilding: Ethics, Power and Responsibility in International Relations (University of Westminster, 9-11 de octubre de 2009), en https://www.westminster. ac.uk/_data/assets/pdf_file/0009/81585/Billerbeck.pdf, consultado el 3.XI.2015; H. ReIcH, “«Local Ownership» in Conflict Transformation Projects Partnership, Participation or Patronage?” (Berghof Occasional Paper, 27), 2006, en http:/ / www.berghof-foundation.org/fileadmin/redakti on/Publications/Papers/Occasional_Papers/boc27e.pdf, consultado el 3.XI.2015; KoHL, informe citado, p. 12.

120 "Security Sector Reform and the Challenges of Ownership", en Mark Sedra (ed.), The Future of Security Sector Reform, Ontario, Centre for International Governance Innovation, 2010, p. 234. 
rio comprender la RSs como opción política, en la medida en que representa intereses de grupos políticos específicos.

En el caso de Guinea-Bisáu, la formulación de la Rss involucró una escasa participación de la sociedad guineana. ${ }^{121}$ Esta realidad, sumada al entorno de gran precariedad de la seguridad humana en Guinea-Bisáu, ${ }^{122}$ pone en duda la estrategia de seguridad del gobierno. De hecho, la seguridad humana en el país enfrentó, en el período analizado, óbices importantes, como el tráfico ilícito y las violaciones de derechos humanos en el país. ${ }^{123}$ De esta manera, el análisis de las esferas política, de desarrollo y de seguridad en Guinea-Bisáu evidenció un ambiente por demás complejo, el cual conformó el telón de fondo de la actuación de la PBC en ese país, asunto que se expondrá en la siguiente sección.

\section{La Comisión PARa la Consolidación de la Paz EN EL ÁMBiTO GUINEANO: ¿CUÁL PAZ?}

La crisis política, social y de seguridad de Guinea-Bisáu propició su inclusión en la agenda de la PBC, tras el consentimiento del Consejo de Seguridad. ${ }^{124}$ El mandato de la PBC en Guinea-Bisáu tuvo los siguientes propósitos: 1) sugerir estrategias para la consolidación de la paz; 2) coordinar actores nacionales e internacionales; 3) recaudar recursos. ${ }^{125}$ Para

${ }^{121}$ KoHL, informe citado, p. 23.

122 PNud, "Human Development Report. New Dimensions of Human Security", 1994, en http://hdr.undp.org/reports/global/1994/en, consultado el 8.VIII.2015. Según la PNUd (ibid., p. 23), la seguridad humana se relaciona con la idea de que el desarrollo humano es esencial para la paz, de manera que si se quiere encauzarlo, se necesitan de estas siete dimensiones: salud, economía, alimentación, medio ambiente, personal, comunidad y política.

${ }^{123}$ UNODC, art. cit., p. 10; LGDH, informe citado y "Guiné-Bissau: 40 anos de impunidade", 2013, en http://www.lgdh.org/p/relatorios.html, consultado el 9.I.2016.

${ }^{124} \mathrm{~A} / \mathrm{RES} / 60 / 180$, doc. cit.

125 Ibid., § 2. 
concretar estas aspiraciones, la PBC desarrollaría cuatro actividades principales: la planificación de la consolidación de la paz en Guinea-Bisáu, no antes de definir los temas prioritarios para el caso; realizar reuniones de trabajo; hacer visitas a Guinea-Bisáu y emitir declaraciones diplomáticas.

Respecto de la primera actividad -la definición de acciones perentorias para posibilitar la consolidación de la paz en el país-, la PBC elaboró un documento denominado "Cuadro Estratégico para la Consolidación de la Paz en Guinea-Bisáu” (en adelante "Cuadro Estratégico"), ${ }^{126}$ el cual consistió en un plan de acción en que se establecieron seis temas primordiales para la consolidación de la paz en este país y que se relacionan con las tres esferas analizadas en el presente trabajo, política, desarrollo y seguridad: 1) elecciones; 2) medidas para mejorar la economía; 3) reforma del sector de seguridad y defensa; 4) fortalecimiento del sector de Justicia, del Estado de Derecho y lucha contra el tráfico de drogas; 5 ) reforma de la administración pública; 6) cuestiones sociales. ${ }^{127}$

La segunda actividad consistió en las reuniones de trabajo de la Configuración Específica y del Grupo sobre Lecciones Aprendidas. Las de aquélla, por un lado, fueron encuentros periódicos con el objetivo de discutir la situación de consolidación de la paz en Guinea-Bisáu; entre 2007 y 2014, se realizaron ocho reuniones de esa naturaleza. ${ }^{128}$ Las de éste, por

${ }^{126} \mathrm{PBC} / 3$ / GNB /3, "Strategic Framework for Peacebuilding in GuineaBissau", 2008, en http://www.un.org/ga/search/view_doc.asp?symbol= PBC/3/GNB/3, consultado el 17.I.2016.

127 Ibid., 13.

${ }^{128}$ Las reuniones de la Configuración Específica fueron las siguientes: $\mathrm{PBC} / 3 / \mathrm{GNB} / \mathrm{SR} .1$, "Summary Record of the Guinea-Bissau Configuration First Meeting”, 2008, en http://www.un.org/ga/search/view_doc. asp?symbol=PBC/3/GNB/SR.1, consultado el 17.I.2016; PBC/2/GNB/SR.2, "Summary Record of the Guinea-Bissau Configuration Second Meeting", 2008, en http://www.un.org/ga/search/view_doc.asp?symbol=PBC/2/ GNB/SR.2, consultado el 17.I.2016; PBC/2/GNB/SR.3, "Summary Record of the Guinea-Bissau Configuration Third Meeting”, 2008, en http:/ /www. un.org/ga/search/view_doc.asp?symbol=PBC/2/GNB/SR.3, consultado el 17.I.2016; PBC/4/GNB/sR.1, "Summary Record of the Guinea-Bissau 
otro, constaron de debates sobre temas comunes a los países de la agenda de la PBC.

La tercera actividad de la PBC se concentró en las misiones de miembros de ésta en Guinea-Bisáu para el planeamiento de tareas con representantes guineanos. Entre 2007 y 2014, se realizaron siete misiones, ${ }^{129}$ con una duración pro-

Configuration First Meeting", 2010, en http://www.un.org/ga/search/ view_doc.asp?symbol=PBC/4/GNB/SR.1, consultado el 17.I.2016; csC, "Chair's Summary of the Informal Meeting of the Guinea-Bissau Configuration”, 2013, en http://www.un.org/en/peacebuilding/cscs/gui/informal/GB\% 20CSM\% 20Chair's\%20Summary\%2010 May2013.pdf, consultado el 17.I.2016; cSC, "Chair's Summary of the Informal Meeting of the Guinea-Bissau Configuration [I]”, 2014, en http://www.un.org/en/ peacebuilding/cscs/gui/informal/Sumary\%20of\%20GB\% 20Configuration\%20meeting\%2010\%20March\%20_final_.pdf, consultado el 17.I.2016; Csc, "Chair's Summary of the Informal Meeting of the Guinea-Bissau Configuration [II]", 2014, en http://www.un.org/en/peacebuilding/cscs/ gui/informal/20140512\%20-\%20PBC\%20Guinea\%20Bissau \% 20Configuration \%20meeting\%20minutes.pdf, consultado el 17.I.2016; csC, "Chair's Summary of the Informal Meeting of the Guinea-Bissau Configuration [III]", 2014, en http://www.un.org/en/peacebuilding/cscs/gui/informal/20140716\%20-\% 20Summary\% 20of\% 20Guinea \% 20Bissau \% 20configuration \%20informal\%20meeting.pdf, consultado el 17.I.2016.

${ }^{129}$ Las referencias de las misiones son: csc, "Summary Notes of the Chair on the Briefing of the Field Mission of H. E. Ms. Maria Luiza R. Viotti", 2008, en http:/ / www.un.org/en/peacebuilding/cscs/gb/pbc_visits/gb_informal_chair_summ_5feb2008.pdf, consultado el 17.I.2016; Csc, "Report of the Mission to Guinea-Bissau of the Peacebuilding Commission Guinea Bissau Configuration”, 2008, en http:/ /www.un.org/en/ peacebuilding/cscs/gb/pbc_visits / chair_visit_gb_10_12_09_08.pdf, consultado el 17.I.2016; csc, "Report of the Chair's Mission to GuineaBissau [I]", 2014, en http://www.un.org/en/peacebuilding/cscs/gui/ pbc_visits/PBC_GB-\%20Report\%20Chair\%20mission \% 2015\%2024\% 20 $01 \% 2014 \% 20$.pdf, consultado el 17.I.2016; csc, "Report of the Chair's Mission to Guinea-Bissau [II]", 2014, en http://www.un.org/en/peacebuilding/cscs/gui/pbc_visits/1401106\%20Amb\%20Patriota_GB $\% 20$ mission\%20report_Oct\%202014\%20-\%20Final.pdf, consultado el 17.I. 2016; PBC/2/GNB/7, "Report of the Mission of the Peacebuilding Commission to Guinea-Bissau", 2008, en http://www.un.org/ga/search/ view_doc.asp?symbol=PBC/2/GNB/7, consultado el 17.I.2016; PBC/2/ GNB/6, "Report of the Mission of the Peacebuilding Commission to Gui- 
medio de tres días, en las cuales los participantes de la Configuración Específica mantuvieron contacto, principalmente, con representantes del gobierno. ${ }^{130}$ Finalmente, el cuarto tipo de actividad desarrollado fueron las declaraciones diplomáticas, que demostraron el posicionamiento oficial de la $\mathrm{PBC}$ respecto de ciertos acontecimientos en Guinea-Bisáu. ${ }^{131}$

nea-Bissau", 2008, en http://www.un.org/ga/search/view_doc.asp?sym bol=PBC/2/GNB/6, consultado el 17.I.2016; PBC/4/GNB/4, "Report of the Peacebuilding Commission Mission to Guinea-Bissau, 16-21 January 2010", 2010, en http://www.un.org/ga/search/view_doc.asp?symbol= $\mathrm{PBC} / 4 / \mathrm{GNB} / 4$, consultado el 17.I.2016.

130 Apenas tres organizaciones de la sociedad civil se citaron en los documentos de la PBC entre 2007 y 2014: Voz di Paz (PBC/4/GNB/1, doc. cit., $\S 51$; csc, "Chair's Summary of the Informal Meeting of the GuineaBissau Configuration”, 2014a, p. 5), AmaE (Asociación de Mujeres en Actividad Económica) y la Comisión de las Mujeres Trabajadoras (CMNT) (id.).

131 Las referencias de las declaraciones son: $\mathrm{PBC} / 3 / \mathrm{GNB} / 5$, "Statement by the Chair of the Peacebuilding Commission's Configuration on Guinea-Bissau”, 2009, en http://www.un.org/ga/search/view_doc.asp? symbol=PBC/3/GNB/5, consultado el 17.I.2016; PBC/3/GNB/6, "Statement by the Chair of the Guinea-Bissau Configuration of the Peacebuilding Commission", 2009, en http://www.un.org/ga/search/view_doc. asp? symbol=PBC/3/GNB/6, consultado el 17.I.2016. También, a cargo de M. L. Viotti, "Statement by the Chair of the Peacebuilding Commission's Country-Specific Configuration on Guinea-Bissau”, 2012, en http:/ /www. un.org/en/peacebuilding/pdf/Statement \% 20Chair\% 20CSM \% 20 GB,\%2013\%20April\%202012\%20\%20.pdf, consultado el 17.I.2016; "Statement of the Chair of the Guinea-Bissau Configuration of the PBC to the Security Council [I]", 2012, en http://www.un.org/en/peacebuilding/ pdf $/ 2012 \%$ 204\% 2018\% 20Chair\%20Statement \% 20SC\% 20briefing\% 20 19\%20April\%202012.pdf, consultado el 17.I.2016; "Statement of the Chair of the Guinea-Bissau Configuration of the PBC to the Security Council [II]", 2012, en http://www.un.org/en/peacebuilding/pdf/statement _chair_sc_brief_gb_2012_7_26.pdf, consultado el 17.I.2016; "Report of the Chairpersonship of Ambassador Maria Luiza Ribeiro Viotti as Chair of the Guinea-Bissau Configuration (December 2007 - May 2013)", 2013, en http://www.un.org/en/peacebuilding/cscs/gui/key_docs/2013\%20 $6 \% 203 \%$ 20GB \% 20CSM\% 20Chair's \% 20Final\% 20repor \% 20December\%202007\% 20to\%20May\%202013.pdf, consultado el 17.I.2016. Asimismo, véase A. de A. Patriota, "Statement of the Chair of the Guinea-Bissau Configuration of the PBC to the Security Council", 2014, en http://www. 
De manera esquemática, estos cuatro grupos de actividades de la PвC pueden distribuirse en tres cortes temporales: de 2007 a 2012; de 2012 a 2014; y a partir de 2014. El primero se caracterizó por el compromiso inicial de la $\mathrm{PBC}$ en Guinea-Bisáu, período en que se llevaron a cabo las primeras misiones oficiales de los miembros de esta Comisión en el país y el planeamiento de sus actividades. El segundo se caracteriza, en cambio, por la reducción significativa de la actuación de la PBC. Según Viotti, ${ }^{132}$ a causa del golpe militar de 2012, la PBC perdió, aunque temporalmente, sus interlocutores en el país. En consecuencia, sólo con las elecciones de 2014, que establecen el inicio del tercer corte temporal, la PBC pudo restablecer su compromiso en el ámbito guineano.

A lo largo del período analizado, la PBC actuó en conjunto con el Fondo para la Consolidación de la Paz, creado en 2005 con miras a coordinar la asignación de recursos financieros en los procesos de consolidación de la paz. Entre 2007 y 2014, el Fondo aprobó US\$ 27.1 millones para proyectos en Guinea-Bisáu, los cuales se destinaron a los tres ejes de actuación comentados en este trabajo: $50 \%$ al área de Rss; $35 \%$ al desarrollo y $15 \%$ a la política. ${ }^{133}$

Así, para comprender la naturaleza de las acciones de la PBC y del Fondo para la Consolidación de la Paz en Guinea-

un.org/en/peacebuilding/pdf/disc\%20GB \% 20CSNU\% 2026\%20Fev. pdf, consultado el 17.I.2016, y, de la csc, los siguientes documentos: "Press Statement of the Guinea-Bissau Configuration", 2014, en http://www. un.org/en/peacebuilding/pdf/upcomingelections1aprilstatement.pdf, consultado el 17.I.2016; "Guinea-Bissau Configuration of the Peacebuilding Commission Elections - 2nd round (18 May 2014) - Press Statement", 2014, en http://www.un.org/en/peacebuilding/pdf/GB $\% 20$ Press $\% 20$ Statement\%20Round\%20Two\%20_FINAL_\%2014\%20May\%202014.pdf, consultado el 17.I.2016; "Statement by the Chair of the Guinea-Bissau Configuration of the Peacebuilding Commission", 2014, en http://www. un.org/en/peacebuilding/pdf/GB\% 20-\% 20disc\%20GB\% 20Chair19mai\%20-\%20FINAL.pdf, consultado el 17.I.2016.

132 "Report of the Chairpersonship...", p. 9.

133 MPTF, “Guinea-Bissau Country Factsheet", 2015, en http://mptf. undp.org/factsheet/country/GNB, consultado el 21.XII.2015. 
Bisáu, este apartado divide el análisis según las categorías de estudio, atinentes a política, desarrollo y seguridad. Dicho esto, el análisis de la actuación de la PBC se inicia por la primera categoría recién apuntada, la política, en la cual la promoción de democracias constituyó un elemento estructurante de acción de la PBC en Guinea-Bisáu. En este sentido, la lógica democrática de la PBC y del Fondo para la Consolidación de la Paz se manifestó por medio de proyectos que buscaban apoyar los procesos electorales en GuineaBisáu y, en menor medida, promover otras formas de diálogo político, como debates abiertos a la participación popular. El presupuesto del Fondo para proyectos en el ámbito político fue de US\$4 279410.88 , cantidad de la cual se destinó el $65.6 \%$ al tema de las elecciones ${ }^{134}$ y el $34.3 \%$ a otras formas de diálogo político. ${ }^{135}$ Entre 2007 y 2014, el monto efectivamente empleado en elecciones, US\$2 624 154.07, superó

${ }^{134}$ Los proyectos relativos al tema de las elecciones fueron los siguientes: PBF/GNB/A-1, "Project 66671: Support Project to Guinea Bissau's Electoral Cycle, Phase I", 2008, en http://mptf.undp.org/factsheet/project /00066671, consultado el 18.I.2016; PBF/IRF-82, "Project 89351: Support to the Electoral Commission in Guinea-Bissau", 2014, en http://mptf. undp.org/factsheet/project/00089351, consultado el 18.I.2016; PBF/IRF85, "Project 89713: Strengthening Electoral Security in the Country, Covering All Regions (Guinea-Bissau)", 2014, en http://mptf.undp.org/ factsheet/project/00089713, consultado el 18.I.2016; PBF/IRF-86, "Project 89877: Strenghtening the Media's Watchdog Role during the 2014 Elections in Guinea Bissau", 2014, en http://mptf.undp.org/factsheet/ project/00089877, consultado el 18.I.2016; PBF/IRF-87, "Project 89930: Strengthening Women's participation in the Elections as Candidates, Voters and Monitors (Guinea-Bissau)", 2014, en http://mptf.undp.org/ factsheet/project/00089930, consultado el 18.I.2016.

135 Los proyectos sobre otras formas de diálogo político son: PBF/ GNB/A-2, "Project 80185: Support to National Reconciliation and Political Dialogue", documento del 21 de octubre de 2011, en http://mptf. undp.org/factsheet/project/00080185, consultado el 18.I.2016, y PBF/ IRF-70, "Project 87798: National Commission for Planning and Strategic Coordination (Guinea-Bissau)", documento del 27 de septiembre de 2013, en http://mptf.undp.org/factsheet/project/00087798, consultado el 18.I.2016. 
en gran medida los gastos asignados a otras formas de diálogo político (US\$36 129.66). ${ }^{136}$

En cuanto a los documentos ${ }^{137}$ elaborados por la $\mathrm{PBC}$ para Guinea-Bisáu en el asunto político, se observó que la primera fase de la actuación de la PBC se basó en 1) el realce de las elecciones y en 2) el escaso compromiso con otras formas de diálogo político, a pesar de que la Comisión declarara, reiteradamente, la importancia de la "apropiación local". El primer ítem, el informe "Nota de Contexto sobre las Elecciones Legislativas del 16 de noviembre de 2008", ${ }^{138}$ uno de los primeros documentos redactados por la $\mathrm{PBC}$ a propósito del país, trató el tema de la política poniendo especial atención en la organización de elecciones, no así en otras formas de diálogo político. Además, en 2008, la PBC elaboró el Cuadro Estratégico para la Consolidación de la Paz, en el cual la celebración de las elecciones se destacó como la prioridad determinante para la consolidación de la paz en Guinea-Bisáu. ${ }^{139}$ De este modo, se hizo evidente que el compromiso político de la $\mathrm{PBC}$ en el país tendría como propósito fundamental el establecimiento de un gobierno democráticamente electo. Esta importancia atribuida a las elecciones se dio, sin embargo, en detrimento de un mayor compromiso con la sociedad guineana, lo que puso de manifiesto el hecho de que el vínculo entre ésta y la PBC se limitó a la presencia de algunos representantes de organizaciones de la sociedad civil guineana en sus misiones y visitas. Entre 2008 y 2011, por

136 MPTF, doc. cit.

${ }^{137}$ Los documentos constan de actas de reuniones, informes de visitas y declaraciones diplomáticas, disponibles en la página institucional de la Comisión (PBC, "Guinea Bissau”, 2016, en http:/ / www.un.org/en/peacebuilding/doc_guinea-bissau.shtml, consultado el 12.VII.2016) y en las Referencias bibliográficas.

138 Рвс, "Background Paper on Guinea-Bissau legislative elections on 16 November 2008", en http:/ / www.un.org/en/peacebuilding/cscs/gb/ key_docs/bg_paper_elections_2008.pdf, consultado el 12.VII.2016.

139 Pвс/3/GNB/3, doc. cit., p. 5. 
ejemplo, sólo la organización Voz di Paz tuvo alguna mención, nominal apenas, en los informes de la PBC. ${ }^{140}$

En consecuencia, el segundo período de compromiso de la PBC en Guinea-Bisáu, entre 2012 y 2014, estuvo marcado por su escasa actuación, toda vez que, retirada la vía electoral en razón del golpe de 2012, se vio destituida de su principal forma de contacto político con el país. Por lo tanto, sus declaraciones diplomáticas tuvieron por entonces poca fuerza propositiva y se volcaron, preponderantemente, en condenar el golpe militar en el país, en relatar la situación de Guinea-Bisáu ante el Consejo de Seguridad y la Asamblea General y en recomendar al gobierno de Guinea-Bisáu que hiciera esfuerzos para restablecer el diálogo y consolidar la paz en su territorio. ${ }^{141}$ Así, pues, al concentrar sus esfuerzos políticos en el ámbito de la promoción de elecciones, la Comisión tuvo un compromiso limitado en cuanto a otras formas de diálogo político para con la sociedad guineana. De esta forma, se reforzó el argumento de que los proyectos de la PBC en el plano político tuvieron un sesgo de paz liberal, pues el fomento a la democracia por la vía electoral constituyó su principal medio de actuación en el ámbito político de Guinea-Bisáu.

Después de efectuarse las elecciones de 2014, que supusieron el fin del golpe militar, se inauguró el tercer período de compromiso de la PBC, la cual, sin embargo, no renovó sus iniciativas para impulsar el diálogo que la aproximara a la sociedad guineana. La propuesta del presidente de la Configuración para Guinea-Bisáu, el embajador Antonio de Aguiar Patriota, corroboró este argumento después de las elecciones de 2014 al sostener que la promoción del diálogo político debía darse a propósito de un proceso claro

$140 \mathrm{PBC} / 4 / \mathrm{GNB} / 1$, doc. cit., $\$ 51$.

141 Véase Viotri, "Statement by the Chair of the Peacebuilding..."; "Statement of the Chair of the Guinea-Bissau Configuration of the PBC to the Security Council [I]"; "Statement of the Chair of the Guinea-Bissau Configuration of the PBC to the Security Council [II]"; $y$ "Report of the Chairpersonship...". 
de Rss. ${ }^{142}$ Así, la única propuesta de diálogo político se formuló a partir de un tema externamente definido, la Rss, pero se dejó de lado el esbozo de una que procurase comprender las razones de los conflictos políticos imperantes en la sociedad guineana. A causa de la estrechez de miras, entre 2007 y 2014, la Comisión asumió que el celebrar comicios sería la solución política para la pacificación de las crisis de GuineaBisáu. Por lo tanto, en el ámbito político, la presunción de la PBC en cuanto a la universalidad de la consolidación de la paz liberal provocó su distanciamiento con las necesidades auténticas del pueblo guineano.

En la segunda categoría de análisis de este estudio, el desarrollo, ese alejamiento entre la PBC y la sociedad local fue, también, puesto en evidencia. En lo que hace al Fondo para la Consolidación de la Paz, el desarrollo fue tema de poca relevancia, pues sólo cuatro proyectos, de un total de veinte, se refirieron al asunto: 1) "Empleos para jóvenes y mujeres en Guinea-Bisáu”;143 2) “Apoyo a la creación de empleo y de renta en Guinea-Bisáu"; 144 3) "Impacto multinivel para dar poder económico a las mujeres en Guinea-Bisáu"; 145 4) "Entrenamiento y empleo de jóvenes profesionistas". ${ }^{146} \mathrm{El}$ propósito de todos ellos, según puede observarse, reza muy

142 Csc, "Chair's Summary of the Informal Meeting of the GuineaBissau Configuration [II]", p. 4.

143 PBf/IRF-76, "Project 88453: Labor-Intensive Employment for Youth and Women in Lead-Up to and Immediate Post-Electoral Period in Guinea Bissau", 2013, en http:/ / mptf.undp.org/factsheet/project/00088 453, consultado el 18.I.2016.

${ }^{144} \mathrm{PbF} / \mathrm{GNB} / \mathrm{D}-2$, "Project 80725: Appui à la création d'emplois et de revenus en Guinée Bissau”, 2012, en http://mptf.undp.org/factsheet/ project/00080725, consultado el 18.I.2016.

145 PbF/IRf-50, "Project 82143: Quick and Multilevel Impact for Women's Economic Empowerment and Improvement of Working Conditions in Guinea-Bissau", documento del 30 de junio de 2015, en http:/ / mptf.undp.org/factsheet/project/00082143, consultado el 18.I.2016.

${ }^{146} \mathrm{PBF} / \mathrm{GNB} / \mathrm{D}-1$, "Project 66674: Youth Professional Training and Employment", informe final del proyecto, 31 de diciembre de 2011, en http:/ / mptf.undp.org/factsheet/project/00066674, consultado el 18.I.2016. 
claramente en el título y reside, también es patente, en ciertos sectores de fuerza laboral desatendidos.

Si bien el primer proyecto propició la creación de aproximadamente 400 empleos en el país, ${ }^{147}$ el segundo y tercero se interrumpieron por el golpe militar de 2012, aunque este último se retomó en 2013 y dio como resultado el mejoramiento de 23 pequeñas y medianas empresas y la legalización de 15 organizaciones de mujeres. ${ }^{148}$ También el cuarto proyecto se concluyó; sin embargo, su informe de evaluación final destacó que el apoyo que había ofrecido la iniciativa de las Naciones Unidas no fue "pertinente", ya que se mostró demasiado "clásico" a causa de su incapacidad para comprender las necesidades del mercado de Guinea-Bisáu. ${ }^{149}$ Por lo tanto, esas evaluaciones dieron testimonio de que tres proyectos se malograron tanto por el golpe militar de 2012 -durante el cual la PBC actuó de manera insuficiente en la promoción del diálogo político-, cuanto por los menguados esfuerzos de los equipos responsables de tales proyectos para discernir los problemas locales.

En el ámbito de desarrollo, se analizaron los documentos que elaboró la PBC, entre 2007 y 2014, respecto de GuineaBisáu, lo cual puso al descubierto propuestas de actuación genéricas y una escasa movilización de recursos. Por ejemplo, la propuesta de estrategias genérica se expuso en el informe de seguimiento del Marco Estratégico de 2008, según el cual la PBC debía "continuar apoyando al gobierno en el tratamiento de los desafíos de los sectores sociales". ${ }^{150}$ Este documento no mencionó, sin embargo, los instrumentos que habrían de utilizarse o, peor aún, las iniciativas prácticas que pondrían en marcha ese apoyo. En el marco de la mo-

147 PBF/IRF-76, doc. cit.

148 PBF/IRF-50, doc. cit., p. 9.

$149 \mathrm{PBF} / \mathrm{GNB} / \mathrm{D}-1$, informe citado, p. 7.

${ }^{150} \mathrm{PBC} / 4 / \mathrm{GNB} / 1 /$ add.1, "Progress Report on the Implementation of the Peacebuilding Strategic Framework for Guinea-Bissau - Addendum", 2010, p. 14, en http://www.un.org/ga/search/view_doc.asp?symbol=PBC/ 4/GNB/1/add.1, consultado el 17.I.2016. 
vilización de recursos, solamente en tres ocasiones, entre 2007 y 2014, la PBC abogó por el apoyo internacional para mejorar el desarrollo en Guinea-Bisáu. ${ }^{151}$ Por ello, los documentos elaborados por la PBC para Guinea-Bisáu a propósito de la categoría correspondiente al desarrollo demostraron su deficiente compromiso en el asunto, el cual, por lo demás, ocupó minúsculo espacio en su agenda.

A diferencia del desarrollo, la Comisión dedicó su atención de manera prioritaria a la Reforma del Sector de Seguridad (Rss). Este hecho quedó demostrado tanto en el marco de los proyectos del Fondo para la Consolidación de la Paz, como en los documentos realizados por la PBC para GuineaBisáu relativos a la Rss. En cuanto al Fondo, el $50 \%$ del presupuesto total para Guinea-Bisáu entre 2007 y 2014, es decir US\$13 455802.00 , se destinó a la Rss, ${ }^{152}$ lo que refuerza el argumento de que esta agenda, delineada preponderantemente por autoridades gubernamentales y apoyada por actores internacionales, adoptó un papel protagónico en detrimento de la agenda de desarrollo, que sólo recibió el 35\% del total de los recursos. Entre 2007 y 2014 se realizaron siete proyectos de RSS: 1) "Proyecto para la rehabilitación de cuarteles militares", que buscaba, evidentemente, mejorar las instalaciones militares, ${ }^{153} 2$ ) "Rehabilitación de prisiones y entrenamiento de equipos de penitenciarías”, cuyo propósito era renovar la infraestructura penitenciaria y perfeccionar el adiestramiento de sus agentes, ${ }^{154}$ 3) "Evaluación para el entrenamiento de

151 Id.; Viотti, "Report of the Chairpersonship...", p. 5.

152 MPTF, doc. cit.

153 PвF/GNB/в-2, "Project 66673: Project for the Rehabilitation of Military Barracks [I]", documento del 31 de diciembre de 2009, en http://mptf.undp.org/factsheet/project/00066673, consultado el 18.I.2 016; PBF/GNB/B-2, "Project 66673: Project for the Rehabilitation of Military Barracks [II]", documento del 31 de diciembre de 2011, en http:/ / mptf.undp.org/factsheet/project/00066673, consultado el 18.I.2016.

${ }^{154}$ PBF/GNB/B-1, "Project 66672: Rehabilitation of Selected Prisons", documento del 31 de agosto de 2011, en http://mptf.undp.org/factsheet/project/00066672, consultado el 18.I.2016. 
militares", que identificaría las demandas de este tipo de capacitación;155 4) “Apoyo para la organización de Conferencias Nacionales en Defensa y Seguridad”, el cual ayudaría al gobierno en la realización de encuentros entre miembros del sector de seguridad sobre Rss; ${ }^{156}$ 5) "Apoyo a la Reforma del Sector de Defensa y Seguridad", destinado a contribuir con el gobierno en su Estrategia de Rss; ${ }^{157}$ 6) "Fortalecimiento de la Seguridad Interna y del Sistema de Justicia”, ideado para mejorar el acceso de los guineanos al Poder Judicial; ${ }^{158}$ 7) "Fortalecimiento de la Seguridad y de la Estabilidad en Guinea-Bisáu”, que tendría por propósito desarrollar los sistemas de información contra el tráfico de drogas. ${ }^{159}$

Los proyectos llevan a interrogarse acerca de si la Rss, identificada con el rótulo de prioritaria por la PBC, corresponde a las necesidades auténticas y verificables en la sociedad guineana. Estudios hay que hacen catálogo de los padecimientos en este país, como, por ejemplo, el precario sistema de seguridad pública guineano a lo largo del período analizado; ${ }^{160}$ el complejo problema que representa el

${ }^{155}$ PвF/GNB / в-3, "Project 75670: Feasibility Study in Guinea Bissau", 2010, en http://mptf.undp.org/factsheet/project/00075670, consultado el 18.I.2016.

${ }^{156}$ PbF/GNB/B-4, "Project 77439: Support for the Preparatory Conferences for the Defense and Security Sectors within the National Conference Process", 2011, en http://mptf.undp.org/factsheet/project/000774 39, consultado el 18.I.2016.

${ }_{157}$ PBf/GNB/в-5, "Project 80454: Military SSR- Support to Security and Defense Sector Reform and Socio-Economic Reintegration”, 2011, en http://mptf.undp.org/factsheet/project/00080454, consultado el 18.I.2016.

158 PBf/Gnв/в-6, "Project 80477: Strengthening Internal Security and Criminal Justice Systems in Guinea-Bissau", 2011, en http://mptf. undp.org/factsheet/project/00080477, consultado el 18.I.2016.

${ }^{159}$ PBf/IRF-93, "Project 91570: Strengthening Security and Stability in Guinea-Bissau", 2014, en http://mptf.undp.org/factsheet/project/0 0091570, consultado el 18.I.2016.

${ }^{160}$ Kohl, informe citado; Crisis Group, "Para além dos compromissos..." y "La réforme du secteur de la sécurité en Guinée-Bissau...". 
tráfico de drogas; ${ }^{161}$ o el historial de violaciones a los derechos humanos. ${ }^{162}$ Además de esto, al menos dos proyectos realizados mediante el Fondo para la Consolidación de la Paz ofrecen información que desdice lo que se había diseñado en ellos. Uno es el que planeaba rehabilitar los cuarteles militares, ${ }^{163}$ cuyo informe destacó que no se llevaron a cabo las investigaciones necesarias para percatarse de los requisitos técnicos del proyecto. Por ello, y porque se reveló además que su presupuesto era insuficiente para alcanzar el objetivo inicialmente previsto, su planificación se tildó de "poco realista". ${ }^{164}$ El otro proyecto, "Rehabilitación de prisiones y entrenamiento de equipos de penitenciarías", previó la construcción de cuatro cárceles en Guinea-Bisáu. ${ }^{165}$ Sin embargo, al igual que sucedió con el anterior, la falta de una evaluación preliminar acerca de las necesidades técnicas y el reducido presupuesto hicieron que se lo calificara con idéntico título; a la postre, sólo se construyeron dos prisiones. ${ }^{166}$ Aunque los proyectos se hubieran concluido, es necesario preguntarse si el perfeccionamiento de la infraestructura militar propiciaría, per se, la consolidación de la paz. En el caso del "Proyecto para la Rehabilitación de los cuarteles militares", por ejemplo, se partió de la premisa de que invertir en el mejoramiento de las dependencias militares traería estabilidad a las Fuerzas Armadas y, por consiguiente, tendría resultados positivos para la política guineana. ${ }^{167}$ Tal premisa, sin embargo, andaba del todo descaminada, pues, menos de un año después de concluido el proyecto, sobrevino el golpe

161 UnOdC, art. cit.; O'REAGAN y Thompson, informe citado.

162 LGDH, inf. cit. y art. cit.

163 PBF/GNB/B-2, "Project 66673: Project for the Rehabilitation of Military Barracks [I]".

164 PBF/GNB/B-2, "Project 66673: Project for the Rehabilitation of Military Barracks [II]", p. 6.

$165 \mathrm{PBF} / \mathrm{GNB} / \mathrm{B}-1$, doc. cit.

166 Ibid., p. 8.

$167 \mathrm{PBF} / \mathrm{GNB} / \mathrm{B}-2$, "Project 66673: Project for the Rehabilitation of Military Barracks [I]”, p. 2. 
militar de 2012. Además, los proyectos nunca contemplaron en qué medida las iniciativas esbozadas proporcionarían mejores condiciones de seguridad a la población, ${ }^{168}$ para lo cual el desarrollo se torna fundamental. Así, pues, es posible afirmar que no hubo correspondencia entre las acciones de la PBC y la realidad inmanente a la sociedad guineana, ya que los proyectos de la Rss no ofrecieron evidencias de poderse adaptar a las necesidades de seguridad humana de Guinea-Bisáu.

Esta predilección de la PBC por la Rss también aparece en documentos como el "Informe de Revisión del Marco Estratégico de 2008 "169 y en las recomendaciones de la PBC al gobierno guineano. ${ }^{170}$ En el primero, la PBC sugirió a Guinea-Bisáu que la Rss debería ser el tema prioritario para la consolidación de la paz. ${ }^{171}$ Además, recomendó al gobierno de este país que la movilización de recursos tendría que dar prioridad a los fondos de jubilación para los militares, con el fin de hacer viable la Rss. ${ }^{172}$

A causa de la eminente prioridad conferida por la PBC a la Rss, se vuelve necesario cuestionar ese presupuesto, sobre todo cuando el análisis de la encuesta llevada a cabo entre 2007 y 2009 por la organización Voz di Paz da pie para ello. ${ }^{173}$

168 PNUD, informe citado.

$169 \mathrm{PBC} / 4 / \mathrm{GNB} / 1$, doc. cit.

170 Ibid., p. 11; PBC/4/GNB/3, "Conclusions and Recommendations of the First Review of the Strategic Framework for Peacebuilding in Guinea-Bissau", 2010, p. 2, en http://www.un.org/ga/search/view_doc. asp?symbol=PBC/4/GNB/3, consultado el 17.I.2016;

$171 \mathrm{PBC} / 4 / \mathrm{GNB} / 1$, doc. cit., p. 10.

172 Ibid., p. 11. Esta prioridad franca conferida por la $\mathrm{PBC}$ a los fondos de jubilación remite al diagnóstico de O'REagan y Thompson (inf. cit., p. 7), quienes afirman que las Fuerzas Armadas eran una institución sobrevalorada, pues sería necesario reunir recursos que permitieran pagar los costos de las jubilaciones de sus miembros.

${ }^{173}$ La Voz di Paz es una organización no gubernamental de GuineaBisáu que tiene como propósito apoyar a actores locales, entre ellos autoridades nacionales y regionales, que actúen en favor de la consolidación de la paz y de la prevención de conflictos (Voz di Paz, "As causas profun- 
La investigación tenía por propósito identificar la percepción de la población de Guinea-Bisáu sobre las causas de los conflictos nacionales. Según los participantes de la encuesta, ${ }^{174}$ había cuatro causas principales: el debilitamiento del Estado, la pobreza, la mala administración de la justicia y el tribalismo. ${ }^{175}$ Para la población, el debilitamiento del Estado podía verificarse por la precariedad de la infraestructura pública, como puertos y carreteras nacionales. ${ }^{176} \mathrm{El}$ segundo factor más recurrente en las respuestas de los encuestados fue la pobreza, la cual se identificó como una de las causas más determinantes de los conflictos en el país. ${ }^{177}$ El tercer aspecto con mayor coincidencia entre las personas interrogadas fue el del acceso a la justicia, limitado en razón de la corrupción y de la falta de funcionarios capacitados. ${ }^{178} \mathrm{El}$ cuarto elemento en que se insistió, el tribalismo, es el aprovechamiento de las diferencias sociales para promover intereses políticos. ${ }^{179}$ De hecho, según Ferreira, el tribalismo contribuyó a que se diera la guerra civil de 1998, pues disputas políticas provocaron polarizaciones en la sociedad respecto de la etnia Balanta. ${ }^{180}$ Por lo tanto, la investigación que realizó la ong Voz di Paz reunió un repertorio más completo de las causas para los conflictos guineanos. Considerando la defensa de la apropiación local hecha por la PBC, ${ }^{181}$ se torna fundamental que ésta se proponga comprender las diferentes perspectivas que brin-

das de conflitos: a voz do povo", 2010, p. 4, en http://www.interpeace. org/wp-content/uploads/2010/08/2010_GB_Interpeace_Voz_Di_Paz_ The_Voice_Of_The_People_PT.pdf, consultado el 28.IV.2015).

${ }^{174}$ La entrevista se llevó a cabo con 3000 personas de los 38 sectores administrativos del país. Entre los participantes, había representantes locales de la sociedad civil, del Estado, de instituciones privadas, tradicionales y religiosas, y de radios comunitarias (ibid., p. 12).

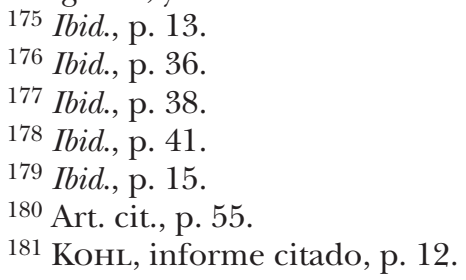


dan fuentes diversas, para superar su visión predominantemente vinculada a autoridades gubernamentales y a procesos electorales.

Con base en lo anterior, es posible afirmar que la paz que procuró la PBC en el ámbito de Guinea-Bisáu a lo largo del período analizado tiene una serie de limitantes: 1) políticas (baste recordar los golpes militares que se dieron a pesar del compromiso de la PBC en la organización de elecciones); 2) de desarrollo (evidenciada por el significativo aumento de la pobreza extrema en el país entre 2010 y 2013) $;^{182} 3$ ) de seguridad (habida cuenta del aumento del tráfico ilícito internacional, ${ }^{183}$ de la precariedad del acceso al sistema de Justicia y de la situación de derechos humanos en el país) ${ }^{184}$

Así, pues, el presente apartado evidenció que el sesgo universalizante de la consolidación de la paz liberal, el cual guió la actuación de la PBC, concedió a la Rss la categoría de prioridad eminente en el marco de las iniciativas de esa Comisión en Guinea-Bisáu. Sin embargo, esta calidad de preeminencia promovió la divergencia entre las iniciativas apoyadas por la PBC y las necesidades de seguridad de la sociedad guineana, en la medida en que la población aún enfrenta el tráfico de drogas y las dificultades para acceder a servicios legales, entre otras cuestiones de seguridad, y en correspondencia con el hecho de que las iniciativas de la PBC y del Fondo se elaboraron sin que hubiera detenidos estudios de evaluación de las necesidades auténticas de la población guineana en lo que toca a materia de seguridad. De esta forma, el relativo distanciamiento de la PBC de las necesida-

182 Banco Mundial, “Terra Ranca! Um novo começo”, 2015, en http:/ / documents.worldbank.org/curated/en/2015/01/24046767/guinea-bissau-country-economic-memorandum-terra-ranca-fresh-start-vol-2-guinebissau-memorando-economico-pais-terra-ranca-um-novo-comeco-relatorio-principal, consultado el 9.I.2016.

183 UnODC, art. cit., p. 10; O'REAGAN y ThOMPson, informe citado, p. 3.

${ }^{184}$ LGDH, art. cit. 
des de Guinea-Bisáu hizo que la iniciativa adoptada con carácter de prioridad, la Rss, no haya sido suficiente para llevar a la población de ese país la paz positiva, la cual, según Johan Galtung, acabaría no sólo con la violencia directa, sino también con la violencia estructural. ${ }^{185}$

Por lo tanto, la aproximación de la PBC a la realidad local no es, per se, condición sine qua non para superar las limitantes, sino condición ineludible para que la estrategia de la PBC pueda mantenerse continuamente organizada para responder a los desafíos del proceso de consolidación de la paz. La complejidad que supone este proceso y la heterogeneidad de las necesidades sociales nacionales exigen una comprensión en profundidad de los problemas del país, lo que requiere de la PBC no sólo involucrarse discursivamente con la apropiación local, sino el esfuerzo permanente para entender la realidad de los guineanos. Un enfoque que se base en la asunción de la universalidad de la paz liberal se revela incapaz de discernimientos, maniobra que precisa, sobre todo, de avivar el diálogo con la sociedad local.

\section{Conclusiones}

Nuestro estudio se ha concentrado en la actuación de la Comisión para la Consolidación de la Paz en Guinea-Bisáu entre 2007 y 2014. Aunque la PBC ha intentado cumplir su mandato en Guinea-Bisáu, su actuación ha adolecido de limitantes en lo que concierne a política, desarrollo y seguridad. En el ámbito político, el camino hacia la consolidación de la paz democrática y liberal emprendido por la Comisión permitió a ésta su aproximación, principalmente, con las autoridades gubernamentales guineanas, pero no necesariamente con los demás grupos sociales del país. De hecho, aunque la historia política de Guinea-Bisáu se ha caracterizado por complejas crisis políticas, las cuales involucraron diversos actores nacio-

${ }^{185}$ Art. cit., pp. 173 y 183. 
nales, como políticos, militares y representantes de otras capas de la sociedad, los esfuerzos de la PBC tuvieron como propósito esencial, ante todo, 1) la interlocución con las autoridades nacionales y 2) el diálogo político mediante la organización de elecciones, con lo cual relegó a segundo plano otros métodos que le aseguraran su cercanía con la población, tales como el debate y la investigación de campo sobre los problemas locales. Así, pues, al reproducir el paradigma de la paz liberal en Guinea-Bisáu, la PBC demostró desidia y fragilidad respecto de la promoción de un diálogo político que procurara con mayor insistencia la pluralidad y condujera a vislumbrar los conflictos presentes en la realidad de los diferentes sectores sociales del país.

Así, aunque las elecciones de 2014 hayan establecido el final del golpe militar iniciado en 2012, la crisis institucional persiste en el país. Según las Naciones Unidas, hasta diciembre de 2017, Guinea-Bisáu no contaba con un gobierno funcional en razón de conflictos entre el presidente José Mario Vaz y su partido, el PAIGC. A causa de estos desencuentros, cinco primeros ministros estuvieron en el poder entre junio de 2014 y noviembre de 2016. ${ }^{186}$ La PBC, en ese entorno, se limitó a emitir declaraciones de apoyo al Acuerdo de Conakry, de 2016, cuyos propósitos son la promoción del diálogo entre las partes en conflicto y el establecimiento de reformas en los poderes ejecutivo, legislativo, judicial y en el sector de seguridad. ${ }^{187}$ De esta forma, la PBC mostró no tener mucha comprensión del escenario político de Guinea-Bisáu, cuyas crisis ocurrieron a pesar de la actuación de la PBC en la organización de elecciones. Éstas, aunque pueden comportar beneficios a la población por constituir un importante medio de participación de la sociedad en la política, no agotan las formas con las cuales la comunidad enuncia sus anhelos. Por lo tanto, según hemos venido reiterando, se debe idear otros

186 Freedom House, Freedom in the World, Guinea-Bisáu, 2017, en http:/ / www.refworld.org/docid/59c102f910.html, consultado el 8.I.2018. ${ }^{187} I d$. 
procedimientos que estimulen el diálogo político y que permitan penetrar en el conocimiento de la realidad de este país.

Por lo que hace al desarrollo, se evidenció que el intrincado ámbito social y económico en que se desenvuelve GuineaBisáu no se alteró a lo largo del período analizado y que la PBC hizo poco para desenmarañarlo. Como se vio en el tercer apartado de este artículo, el diálogo entre la PBC y el ámbito de desarrollo en Guinea-Bisáu se caracterizó por la superficialidad, toda vez que 1) los recursos destinados a llevar a cabo los proyectos del Fondo para la Consolidación de la Paz fueron insuficientes, y 2) las propuestas para impulsar el desarrollo, sumamente imprecisas, a tal grado que su esbozo reveló la incapacidad de la Comisión para tratar con las graves carencias del país en lo que al asunto se refiere. En 2016, por ejemplo, el $30.5 \%$ de la población rural guineana se encontraba en situación de inseguridad alimentaria, 10.5\% más que el año anterior. Este incremento se dio a pesar del aumento del Producto Interno Bruto de Guinea-Bisáu en 2016 respecto de 2015, lo que demuestra que para comprender la cuestión del desarrollo se debe considerar las múltiples interacciones que hay entre las esferas política, económica y social. Así, pues, en términos de desarrollo, el presupuesto de universalidad de la paz liberal, que orientó la actuación de la PBC, provocó que ésta se distanciara de la realidad efectivamente palpable en el terreno guineano.

Por último, se verificó que la categoría atinente a la seguridad, vinculada por la PBC a la Reforma del Sector de Seguridad, fue el tema que recibió mayor atención por parte de la Comisión. Al no buscar comprender de forma integral las causas de los conflictos de Guinea-Bisáu, la PBC adoptó como prioridad las demandas de la cúpula gubernamental en lo que concierne a la Rss. De este modo, la "apropiación local", defendida por la PBC, en la práctica se concentró en aproximarse a las autoridades gubernamentales del país. Si, por un lado, ello permitió alguna especie de contacto con la sociedad, por otro la limitó en gran medida, pues ofreció pocas oportunidades a la PBC de oír las demandas de los integran- 
tes de los diversos sectores sociales y de debatir los problemas nacionales desde una perspectiva pluralista. De hecho, según la Amnistía Internacional, más allá de la cuestión del tráfico internacional ilícito, persisten, en el país, otros problemas de seguridad, como los relacionados con el sistema judicial, el cual presenta casos de corrupción y faltas al no seguir el debido proceso legal. ${ }^{188}$

Por lo tanto, aunque la PBC ha hecho el esfuerzo por cumplir su mandato de consolidar la paz en Guinea-Bisáu, su estrategia en el país reveló una actuación por demás endeble en lo que toca a política, desarrollo y seguridad, elementos fundamentales si se quiere consolidar verdaderamente la paz. Así, al proponerse tomar como guía una tendencia universalizante de paz liberal, la actuación de la PBC contribuyó mínimamente a materializar la paz positiva en el país, ${ }^{189}$ si se tiene en cuenta los complejos obstáculos de las tres categorías analizadas en el presente artículo. Aunque esta Comisión se fundó para avanzar en la agenda de consolidación de la paz, se comprueba que su rationale debe considerarse pieza fundamental de análisis para entender las inconsistencias de su intervención en los problemas de corte político, de seguridad y desarrollo de la sociedad de Guinea-Bisáu.

Trad. de Jesús Jorge Valenzuela

\section{REFERENCIAS BIBLIOGRÁFICAS}

Amnistía Internacional, “Amnesty International Report 2016/17 Guinea-Bissau”, 2017, en http://www.refworld.org/docid/58b 033f5a.html

Banco Mundial, “Terra Ranca! Um novo começo”, 2015, en http:/ / documents.worldbank.org/curated/en/2015/01/24046767/

188 “Amnesty International Report 2016/17 - Guinea-Bissau”, 2017, en http:/ / www.refworld.org/docid/58b033f5a.html, consultado el 8.I.2018. 189 GaltunG, art. cit. 
guinea-bissau-country-economic-memorandum-terra-rancafresh-start-vol-2-guine-bissau-memorando-economico-pais-terra-ranca-um-novo-comeco-relatorio-principal

Banco Mundial, "World Development Indicators", 2015, en http:/ / wdi.worldbank.org/tables

Blanco, Ramon, "Del mantenimiento de la paz al proceso de formación del Estado: un esbozo de los esfuerzos de la onU para la paz internacional", Foro Internacional, 54 (2014), pp. 266-318.

Blanco, Ramon, "The un Peacebuilding Process: An Analysis of its Shortcomings in Timor-Leste", Revista Brasileira de Política Internacional, 58 (2015), núm. 1, pp. 42-62.

Blanco, Ramon, "The Brazilian Engagement with Peace Operations: A Critical Analysis", Revista Brasileira de Política Internacional, 60 (2017), núm. 2, pp. 1-20.

Cavalcante, Fernando, Coming into Life: The Concept of Peace Building in the United Nations, from "An Agenda for Peace" to the Peace Building Commission, tesis, Coimbra, Faculdade de Economia da Universidade, 2013.

Cavalcante, Fernando, "Os reflexos da paz liberal na abordagem das Nações Unidas à consolidação da paz na Guiné-Bissau”, Revista Crítica de Ciências Sociais, 2013, núm. 102, pp. 21-42.

Chandler, David, “A Crítica não-crítica da «Paz Liberal»”, Universitas: Relações Internacionais, 11 (2013), núm. 2, pp. 39-51.

Chesterman, Simon, You, The People: The United Nations, Transitional Administration, and State-Building, Oxford, University Press, 2004.

Chesterman, Simon, "Ownership in Theory and in Practice: Transfer of Authority in UN Statebuilding”, Journal of Intervention and Statebuilding, 1 (2007), pp. 3-26.

Crisis Group, "Guinea-Bissau: In Need of a State", informe sobre África núm. 142, 2 de julio de 2008, en http:/ / www.crisisgroup. org/ /media/Files/africa/west-africa/guinea-bissau/GuineaBissau\%20In\%20Need\%20of\%20a\%20State.pdf

Crisis Group, "Para além dos compromissos: as perspectivas de reforma na Guiné-Bissau”, informe sobre África núm. 183, 2012, en http://www.crisisgroup.org/ / media/Files/africa/west-africa/guinea-bissau/Portuguese \% 20translations/183-au-dela- 
des-compromis-les-perspectives-de-reforme-en-guinee-bissau -portuguese

Crisis Group, "La réforme du secteur de la sécurité en Guinée-Bissau: une occasion à saisir", informe sobre África núm. 109, 2015, en http://www.crisisgroup.org/fr/regions/afrique/afriquede-louest/guinee-bissau/b109-security-sector-reform-in-guinea-bissau-an-opportunity-not-to-be-missed.aspx

DuFField, Mark, Global Governance and the New Wars, Londres, Zed Books, 2001.

Ferreira, Patrícia Magalhães, "Guinea-Bissau: Between Conflict and Democracy", African Security Review, 13 (2004), pp. 44-56.

Freedom House, Freedom in the World, Guinea-Bisáu, 2017, en http://www.refworld.org/docid/59c102f910.html

FMI = Fondo Monetario Internacional, "Guiné-Bissau. Temas selecionados", informe núm. 15/195, 2015, en https://www.imf. org/external/lang/portuguese/pubs/ft/SCR/2015/ cr15195p.pdf

Galtung, Johan, "Violence, Peace, and Peace Research", Journal of Peace Research, 6 (1969), pp. 167-191.

Gomes, Áureo T., "Da paz liberal à virada local: avaliando a literatura crítica sobre peacebuilding”, Monções: Revista de Relações Internacionais da UFGD, 2 (2013), núm. 4, pp. 46-76.

Heathershaw, John, "Unpacking the Liberal Peace: The Dividing and Merging of Peacebuilding Discourses", Millennium. Journal of International Studies, 36 (2008), pp. 597-621.

Jenkins, Rob, Peacebuilding. From Concept to Commission, Nueva York, Routledge, 2013.

Kant, Immanuel, Perpetual Peace: A Philosophical Essay, Londres, George Allen \& Unwin Ltd., 1905 [1795].

Kemer, Thaíse, Alexsandro Eugenio Pereira y Ramon Blanco, "A construção da paz em um mundo em transformação: o debate e a crítica sobre o conceito de peacebuilding", Revista de Sociologia e Política, 24 (2016), núm. 60, pp. 137-150.

KoHL, Christoph, "The Reform of Guinea-Bissau's Security Sector: Between Demand and Practice", Peace Research Institute Frankfurt, informe núm. 126, 2014, en http://issat.dcaf.ch/ 
content/download/73329/1247623/file/prif126\%20The\%20

Reform\%20of\%20GB\%20Security\%20Sector_Kohl.pdf

LGDH = Liga Guineense dos Direitos Humanos, "Relatório sobre a

Situação dos Direitos Humanos na Guiné-Bissau 2008/2009",

2008, en http://www.lgdh.org/p/relatorios.html

LGDH = Liga Guineense dos Direitos Humanos, "Guiné-Bissau: 40

anos de impunidade", 2013, en http://www.lgdh.org/p/relatorios.html

Mac Ginty, Roger, International Peacebuilding and Local Resistance: Hybrid Forms of Peace, Nueva York, Palgrave, 2011.

MDG = Millennium Development Goals, "Millenium Development

Goals: Snapshot for Guinea-Bissau", 2015, en http://mdgs. un.org/unsd/mdg/Resources/Static/Products/Progress20 15/Snapshots/GNB.pdf

Мовекк, Eirin, "Security Sector Reform and the Challenges of Ownership”, en Mark Sedra (ed.), The Future of Security Sector Reform, Ontario, Centre for International Governance Innovation, 2010.

Montesquieu, Charles de Secondat, barão de, The Spirit of the Laws, Cambridge, University Press, 2002 [1748].

Neves, Gilda Motta Santos, Comissão das Nações Unidas para Consolidação da Paz: perspectiva brasileira, Brasilia, FUNAG, 2009.

Neves, José, "Ideologia, ciência e povo em Amílcar Cabral”, História, Ciências, Saúde - Manguinhos, 24 (2017), pp. 333-347.

Newman, Edward, Roland Paris y Oliver P. Richmond, "Introduction", en New Perspectives on Liberal Peacebuilding, Tokio-Nueva York-París, United Nations University Press, 2010, pp. 3-25.

OPLOP = Observatório dos Países de Língua Oficial Portuguesa, "Angola oficializa apoio militar para Rss na Guiné-Bissau", 2011, en http://www.oplop.uff.br/boletim/115/angola-oficializa-apoio-militar-para-reforma-no-setor-de-seguranca-defesa -em-guine-bissau

OPLOP = Observatório dos Países de Língua Oficial Portuguesa, "Instabilidade e insegurança: a Guiné-Bissau entre o abril de 2010 e de 2012”, 2013, en http://www.oplop.uff.br/boletim/ 2160/instabilidade-inseguranca-guine-bissau-entre-abril-de2010-de-2012 
O'Reagan, David y Peter Thompson, "Advancing Stability and Reconciliation in Guinea-Bissau: Lessons from Africa's First Narco-State", Washington, D. C., Africa Center for Strategic Studies, informe especial núm. 2, 2013, en http://www.globalinitiative. net/download/drugs/subsaharan-africa/ACSS $\% 20 \% 20$ Advancing $\% 20$ Stability $\% 20$ and $\% 20$ Reconciliation $\% 20$ in $\% 20$ Guinea\%20Bissau\%202013.pdf

Paris, Roland, At War's End: Building Peace After Civil Conflict, Cambridge, University Press, 2004.

PARIs, Roland, "Understanding the «Coordination Problem» in Postwar Statebuilding", en R. Paris y Timothy Sisk (eds.), The Dilemmas of Statebuilding: Confronting the Contradictions of Postwar Peace Operations, Nueva York, Routledge, 2009.

PARIs, Roland, "Saving Liberal Peacebuilding", Review of International Studies, 36 (2010), pp. 337-365.

Pereira, Amilcar Araujo y Paolo Vittoria, A luta pela descolonização e as experiências de alfabetização na Guiné-Bissau: Amilcar Cabral e Paulo Freire, 2012, Estud. hist. (Rio J.), 25 (2012), núm. 50, pp. 291-311.

PerissinotTo, Renato, "Comparação, história e interpretação: Por uma ciência política histórico-interpretativa”, Revista Brasileira de Ciências Sociais, 28 (2013), núm. 83, pp. 151-165.

Pugh, Michael, "Peacekeeping and Critical Theory", en A. Bellamy y P. Williams (eds.), Peace Operations and Global Order, Nueva York, Routledge, 2005.

Pugh, Michael, "The Political Economy of Peacebuilding: A Critical Theory Perspective", International Journal of Peace Studies, 10 (2005), núm. 2, pp. 23-42.

Ramsвотнам, Oliver, "Reflections on un post-settlement peacebuilding”, International Peacekeeping, 7 (2000), pp. 169-189.

Reich, Hannah, “"Local Ownership» in Conflict Transformation Projects Partnership, Participation or Patronage?” (Berghof Occasional Paper, 27), 2006, en http://www.berghof-foundation.org/fileadmin/redaktion/Publications/Papers/Occasional_Papers/boc27e.pdf 
Richmond, Oliver, "The Problem of Peace: Understanding the «Liberal Peace»", Conflict, Security E Development, 6 (2006), pp. 291-314.

Richmond, Oliver, The Transformation of Peace, Nueva York, Palgrave Macmillan, 2007.

Richmond, Oliver, Peace in Advances in Peacebuilding: Critical Developments and Approaches, Londres, Palgrave Macmillan, 2010.

Richmond, Oliver y Jason Franks, Liberal Peace Transitions: Between Statebuilding and Peacebuilding, Edinburgo, Edinburgh University Press, 2009.

Rizzi, Kamila, "A instabilidade contínua na Guiné-Bissau”, Meridiano 47, 11 (2010), núm. 117, pp. 23-26.

Schumpeter, Joseph, "The Sociology of Imperialism”, en Joseph Schumpeter (comp.), Imperialism and Social Classes: Two Essays by Joseph Schumpeter, Cleaveland, Meridian Books, 1966 [1919]. SEcco, Lincoln, "Trinta anos da Revolução dos Cravos", Revista ADUSP, 2004, núm. 33, pp. 6-12, en http://www.adusp.org.br/ files/revistas/33/r33a01.pdf

Simons, Claudia y Francisca Zanker, "Questioning the Local in Peacebuilding”, 2014, en http://www.spp1448.de/fileadmin/ media/galleries/SPP_Administration/Working_Paper_Series/SPP1448_WP10_Simons_Zanker.pdf

SHAw, Mark, "Drug Trafficking in Guinea-Bissau, 1998-2014: The Evolution of an Elite Protection Network", The Journal of Modern African Studies, 53 (2015), pp. 339-364.

TAYlor, Ian, "What Fit for the Liberal Peace in Africa?", Global Society, 21 (2007), pp. 553-566.

TAYlor, Ian, "Liberal Peace, Liberal Imperialism: A Gramscian Critique", en Oliver Richmond (ed.), Palgrave Advances in Peacebuilding: Critical Developments and Approaches, Londres, Palgrave Macmillan, 2010.

UnODC, "Criminalidade Organizada Transnacional na África Ocidental: Avaliação da Ameaça", 2013, en https://www.unodc. org/documents /data-and-analysis/tocta/West_Africa_TOCTA_2013_PT.pdf

Von Billerbeck, Sarah, "Whose Peace? Local Ownership and UN Peacebuilding", investigación presentada en la conferencia 
The Future of Statebuilding: Ethics, Power and Responsibility in International Relations (University of Westminster, 9-11 de octubre de 2009), en https://www.westminster.ac.uk/_data/assets/ pdf_file/0009/81585/Billerbeck.pdf

Voz di Paz, "As causas profundas de conflitos: a voz do povo", 2010, en http:/ / www.interpeace.org/wp-content/uploads/2010/08/ 2010_GB_Interpeace_Voz_Di_Paz_The_Voice_Of_The_People_PT.pdf

Yabi, Gilles O., The Role of ECOWAS in Managing Political Crisis and Conflict: The Cases of Guinea and Guinea-Bissau, trad. de Roger Addah, Abuja, Nigergia, Friedrich-Ebert-Stiftung, 2010, en http://library.fes.de/pdf-files/bueros/nigeria/07448.pdf

\section{Fuentes primarias}

A /47/277, "An Agenda for Peace", 17 de junio de 1992, en http:/ / daccess-dds-y.un.org/doc/UNDOC/GEN/N92/259/61/ PDF/N9225961.pdf?OpenElement

A/59/565, "A More Secure World: Our Shared Responsibility", 2004, en https://www.un.org/ruleoflaw/blog/document/thesecretary-generals-high-level-panel-report-on-threats-challenges-and-change-a-more-secure-world-our-shared-responsibility/ A/RES/60/180, "Resolução da Assembleia Geral", 2005, en http:/ / www.securitycouncilreport.org/atf/cf/\% 7B65BFCF9B-6D 27-4E9C-8CD3-CF6E4FF96FF9\%7D/PBC\%20ARES60180.pdf

CEDEAO, "Regional Defence Chiefs to Meet on Ecowas Mission in Guinea Bissau”, 2013, en http://news.ecowas.int/presseshow. php?nb=144\&lang=en\&annee $=2013$

CSC, "Summary Notes of the Chair on the Briefing of the Field Mission of H. E. Ms. Maria Luiza R. Viotti”, 2008, en http:// www.un.org/en/peacebuilding/cscs/gb/pbc_visits/gb_informal_chair_summ_5feb2008.pdf

CSC, "Report of the Mission to Guinea-Bissau of the Peacebuilding Commission Guinea Bissau Configuration”, 2008, en http:// www.un.org/en/peacebuilding/cscs/gb/pbc_visits/chair_visit_gb_10_12_09_08.pdf 
CSC, "Chair's Summary of the Informal Meeting of the GuineaBissau Configuration”, 2013, en http://www.un.org/en/peacebuilding/cscs/gui/informal/GB\%20CSM\%20Chair's\% 20 Summary\%2010May2013.pdf

CSC, "Chair's Summary of the Informal Meeting of the GuineaBissau Configuration [I]", 2014, en http://www.un.org/en/ peacebuilding/cscs/gui/informal/Sumary $\% 20$ of $\% 20$ GB $\% 20$ Configuration\%20meeting\%2010\%20March\%20_final_.pdf

CSC, "Chair's Summary of the Informal Meeting of the GuineaBissau Configuration [II]", 2014, en http://www.un.org/en/ peacebuilding/cscs/gui/informal/20140512\%20-\% 20 PBC\% 20Guinea \% 20Bissau \% 20Configuration \% 20meeting\%20minutes.pdf

CsC, "Chair's Summary of the Informal Meeting of the GuineaBissau Configuration [III]", 2014, en http://www.un.org/en/ peacebuilding/cscs/gui/informal/20140716\%20-\%20Summary $\% 20$ of $\% 20$ Guinea $\% 20$ Bissau $\% 20$ configuration $\% 20 \mathrm{in}-$ formal $\% 20$ meeting.pdf

CSC, "Report of the Chair's Mission to Guinea-Bissau [I]", 2014, en http://www.un.org/en/peacebuilding/cscs/gui/pbc_visits/ PBC_GB-\% 20Report\% 20Chair\% 20mission \% 2015\% 2024\% 20 $01 \% 2014 \% 20$.pdf

CSC, "Report of the Chair's Mission to Guinea-Bissau [II]", 2014, en http://www.un.org/en/peacebuilding/cscs/gui/pbc_visits $/ 1401106 \% 20 \mathrm{Amb} \%$ 20Patriota_GB\%20mission \%20report_Oct\%202014\%20-\%20Final.pdf, consultado el 17.I.2016. CSC, "Press Statement of the Guinea-Bissau Configuration", 2014, en http://www.un.org/en/peacebuilding/pdf/upcomingelectionslaprilstatement.pdf

csc, "Guinea-Bissau Configuration of the Peacebuilding Commission Elections - 2nd round (18 May 2014) - Press Statement", 2014, en http://www.un.org/en/peacebuilding/pdf/GB\%20 Press \%20Statement \% 20Round\%20Two\%20_FINAL_\% 20 14\%20May\%202014.pdf

CsC, "Statement by the Chair of the Guinea-Bissau Configuration of the Peacebuilding Commission", 2014, en http://www.un. 
org/en/peacebuilding/pdf/GB\%20-\%20disc\%20GB\%20 Chair19mai\%20-\%20FINAL.pdf

EUA, "United States of America vs Jose Americo Bubo Na Tchuto, Papis Djeme and Tchamy Yala", 2015, en http://www.justice. gov/sites/default/files/usao-sdny/legacy/2015/03/25/ U.S. \%20v.\%20Na\%20Tchuto\%20et\%20al\%20Indictment.pdf MPTF, "Guinea-Bissau Country Factsheet", 2015, en http://mptf. undp.org/factsheet/country/GNB

MRE, "Resolução da CPLP sobre a Situação na Guiné-Bissau", 2012, en http://www.itamaraty.gov.br/pt-BR/notas-a-imprensa/3023 -resolucao-da-cplp-sobre-a-situacao-na-guine-bissau

mre, "Comunidade dos Países de Língua Portuguesa", 2016, en http:/ / www.itamaraty.gov.br/pt-BR/politica-externa/mecanismos-inter-regionais/3676-comunidade-dos-paises-de-lingua-portuguesa-cplp

Naciones Unidas, "The United Nations ssR Perspective", 2012, en http://www.un.org/en/events/peacekeepersday/pdf/securityreform.pdf

PAIGC, "História do PAIGC", 2018, en http://www.paigc.gw/historia.html

Patriota, Antônio de A., "Statement of the Chair of the GuineaBissau Configuration of the PBC to the Security Council”, 2014, en http://www.un.org/en/peacebuilding/pdf/disc\%20GB\% 20CSNU $\% 2026 \% 20 \mathrm{Fev} . p d f$

PBC, "Background Paper on Guinea-Bissau legislative elections on 16 November 2008", en http:/ /www.un.org/en/peacebuilding /cscs/gb/key_docs/bg_paper_elections_2008.pdf

PBC, "Guinea Bissau", 2016, en http://www.un.org/en/peacebuilding/doc_guinea-bissau.shtml

$\mathrm{PBC} / 2 / \mathrm{GNB} / 6$, "Report of the Mission of the Peacebuilding Commission to Guinea-Bissau", 2008, en http://www.un.org/ga/ search/view_doc.asp?symbol=PBC/2/GNB/6

$\mathrm{PBC} / 2 / \mathrm{GNB} / 7$, "Report of the Mission of the Peacebuilding Commission to Guinea-Bissau", 2008, en http://www.un.org/ga/ search/view_doc.asp?symbol=PBC/2/GNB/7 
PBC/2/GNB/SR.2, "Summary Record of the Guinea-Bissau Configuration Second Meeting", 2008, en http://www.un.org/ga/ search/view_doc.asp?symbol=PBC/2/GNB/SR.2 $\mathrm{PBC} / 2$ /GNB/sR.3, "Summary Record of the Guinea-Bissau Configuration Third Meeting", 2008, en http://www.un.org/ga/ search/view_doc.asp?symbol=PBC/2/GNB/SR.3 $\mathrm{PBC} / 3 / \mathrm{GNB} / 3$, "Strategic Framework for Peacebuilding in GuineaBissau", 2008, en http://www.un.org/ga/search/view_doc.asp? symbol=PBC $/ 3 / \mathrm{GNB} / 3$

$\mathrm{PBC} / 3 / \mathrm{GNB} / 5$, "Statement by the Chair of the Peacebuilding Commission's Configuration on Guinea-Bissau", 2009, en http:/ / www.un.org/ga/search/view_doc.asp?symbol=PBC /3/GNB/5 $\mathrm{PBC} / 3 / \mathrm{GNB} / 6$, "Statement by the Chair of the Guinea-Bissau Configuration of the Peacebuilding Commission", 2009, en http:/ / www.un.org/ga/search/view_doc.asp?symbol=PBC/3/ $\mathrm{GNB} / 6$

$\mathrm{PBC} / 3$ /GNB/sR.1, "Summary Record of the Guinea-Bissau Configuration First Meeting", 2008, en http://www.un.org/ga/ search/view_doc.asp?symbol=PBC/3/GNB/SR.1

$\mathrm{PBC} / 4 / \mathrm{GNB} / 1$, "Progress Report on the Implementation of the Peacebuilding Strategic Framework for Guinea-Bissau", 2009, en http://www.un.org/ga/search/view_doc.asp?symbol=PBC/ $4 / \mathrm{GNB} / 1$

$\mathrm{PBC} / 4 / \mathrm{GNB} / 1 /$ add.1, "Progress Report on the Implementation of the Peacebuilding Strategic Framework for Guinea-Bissau - Addendum", 2010, en http:/ /www.un.org/ga/search/view_ doc.asp?symbol=PBC/4/GNB/1/add.1

$\mathrm{PBC} / 4 / \mathrm{GNB} / 3$, "Conclusions and Recommendations of the First Review of the Strategic Framework for Peacebuilding in GuineaBissau", 2010, en http://www.un.org/ga/search/view_doc. asp?symbol=PBC/4/GNB $/ 3$

$\mathrm{PBC} / 4 / \mathrm{GNB} / 4$, "Report of the Peacebuilding Commission Mission to Guinea-Bissau, 16-21 January 2010", 2010, en http://www. un.org/ga/search/view_doc.asp?symbol=PBC/4/GNB/4 $\mathrm{PBC} / 4 / \mathrm{GNB} / \mathrm{sR}$.1, "Summary Record of the Guinea-Bissau Configuration First Meeting", 2010, en http:/ /www.un.org/ga/search /view_doc.asp?symbol=PBC/4/GNB/SR.1 
PBF/GNB/A-1, "Project 66671: Support Project to Guinea Bissau's Electoral Cycle, Phase I", 2008, en http:/ / mptf.undp.org/factsheet/project/00066671

PBF/GNB/A-2, "Project 80185: Support to National Reconciliation and Political Dialogue", documento del 21 de octubre de 2011, en http:/ / mptf.undp.org/factsheet/project/00080185 PBF/GNB/B-1, "Project 66672: Rehabilitation of Selected Prisons", documento del 31 de agosto de 2011, en http://mptf.undp. org/factsheet/project/00066672

PBF/GNB/ B-2, "Project 66673: Project for the Rehabilitation of Mi-

litary Barracks [I]", documento del 31 de diciembre de 2009, en http://mptf.undp.org/factsheet/project/00066673

$\mathrm{PBF} / \mathrm{GNB} / \mathrm{B}-2$, "Project 66673: Project for the Rehabilitation of Military Barracks [II]", documento del 31 de diciembre de 2011, en http://mptf.undp.org/factsheet/project/00066673 PBF/GNB/B-3, "Project 75670: Feasibility Study in Guinea Bissau", 2010, en http://mptf.undp.org/factsheet/project/00075670 $\mathrm{PBF} / \mathrm{GNB} / \mathrm{B}-4$, "Project 77439: Support for the Preparatory Conferences for the Defense and Security Sectors within the National Conference Process", 2011, en http://mptf.undp.org/ factsheet/project/00077439

PBF/GNB/ B-5, "Project 80454: Military SSR-Support to Security and

Defense Sector Reform and Socio-Economic Reintegration", 2011, en http://mptf.undp.org/factsheet/project/00080454 PBF/GNB/ B-6, "Project 80477: Strengthening Internal Security and

Criminal Justice Systems in Guinea-Bissau”, 2011, en http:/ / mptf.undp.org/factsheet/project/00080477

PBF/GNB/D-1, "Project 66674: Youth Professional Training and Employment", informe final del proyecto, 31 de diciembre de 2011, en http://mptf.undp.org/factsheet/project/00066674 $\mathrm{PBF} / \mathrm{GNB} / \mathrm{D}-2$, "Project 80725: Appui à la création d'emplois et de revenus en Guinée Bissau”, 2012, en http://mptf.undp.org/ factsheet/project/00080725

PBF/IRF-50, "Project 82143: Quick and Multilevel Impact for Women's Economic Empowerment and Improvement of Working Conditions in Guinea-Bissau", documento del 30 de junio de 2015, en http://mptf.undp.org/factsheet/project/00082143 
PBF/IRF-70, "Project 87798: National Commission for Planning and Strategic Coordination (Guinea-Bissau)", documento del 27 de septiembre de 2013, en http://mptf.undp.org/factsheet/project/00087798

PBF/IRF-76, "Project 88453: Labor-Intensive Employment for Youth and Women in Lead-Up to and Immediate Post-Electoral Period in Guinea Bissau", 2013, en http://mptf.undp.org/ factsheet/project/00088453

PBF/IRF-82, "Project 89351: Support to the Electoral Commission in Guinea-Bissau", 2014, en http://mptf.undp.org/factsheet/ project/00089351

PBF/IRF-85, "Project 89713: Strengthening Electoral Security in the Country, Covering All Regions (Guinea-Bissau)", 2014, en http://mptf.undp.org/factsheet/project/00089713

PBF/IRF-86, "Project 89877: Strenghtening the Media's Watchdog Role during the 2014 Elections in Guinea Bissau", 2014, en http://mptf.undp.org/factsheet/project/00089877

PBF/IRF-87, "Project 89930: Strengthening Women's participation in the Elections as Candidates, Voters and Monitors (GuineaBissau)", 2014, en http://mptf.undp.org/factsheet/project/ 00089930

PBF/IRF-93, "Project 91570: Strengthening Security and Stability in

Guinea-Bissau", 2014, en http:/ / mptf.undp.org/factsheet/project/00091570

PBso, "Mapping of Resources \& Gaps For Peacebuilding in Guinea-Bissau”, 2008, en http://www.un.org/en/peacebuilding/ cscs/gb/key_docs/mapping_resources_2008.pdf PBso, "Peacebuilding: An Orientation", 2010, en http://www. un.org/en/peacebuilding/pbso/pdf/peacebuilding_orientation.pdf

PNUD, "Human Development Report. New Dimensions of Human Security”, 1994, en http:/ /hdr.undp.org/reports/global/1994/ en

PNUD, "Explanatory Note on the 2014 Human Development Report Composite Indices Guinea-Bissau", 2014, en http://hdr. undp.org/sites/all/themes/hdr_theme/country-notes/GNB. pdf 
República da Guiné-Bissau, "Constituição da República da GuinéBissau", 1996, en http://www.anpguinebissau.org/leis/constituicao/constituicaoguine.pdf/view

República da Guiné-Bissau, "Documento de Estratégia Nacional de Redução da Pobreza”, 2005, en http://www.stat-guinebissau.com/denarp/denarp.pdf

República da Guiné-Bissau, "Reestructuring and Modernization of the Defence and Security Sector", 2006, en http://uniogbis. unmissions.org/Portals/unogbis/Articles/SSR\% 20srtategy\% 20document\% 202006.pdf

s/1998/1028, "Letter dated 3 November 1998 from the Permanent Representative of Nigeria to the United Nations Addressed to the President of the Security Council”, 1998, en http://www. un.org/en/ga/search/view_doc.asp?symbol=S/1998/1028

S/RES/1233, "Resolução do Conselho de Segurança", 1999, en http: / / daccess-dds-ny.un.org/doc/UNDOC/GEN/ N99/096/57/PDF/N9909657.pdf?OpenElement

s/1999/232, "Carta do Secretário-Geral ao Presidente do Conselho de Segurança”, 1999, en http:/ /www.securitycouncilreport. org/atf/cf/\% 7B65BFCF9B-6D27-4E9C-8CD3-CF6E4FF96FF9 $\% 7 \mathrm{D} / \mathrm{GB} \% 20 \mathrm{~S} 1999 \% 20232 . p d f$

S/RES/2048, "Resolução do Conselho de Segurança", 2012, en http:/ / www.un.org/ga/search/view_doc.asp?symbol=S / RES/2048(2012)

Unión Africana, "Report of the Chairperson of the Commission on the Situation in Guinea-Bissau", 2014, en http://www.peaceau. org/uploads/auc-report-guinea-bissau-psc-17-06-2014.pdf

Viotti, Maria L., "Statement by the Chair of the Peacebuilding Commission's Country-Specific Configuration on Guinea-Bissau", 2012, en http://www.un.org/en/peacebuilding/pdf/ Statement\% 20Chair\% 20CSM\%20GB,\% 2013\%20April\% 20 $2012 \% 20 \% 20 . p d f$

Viotтi, Maria L., "Statement of the Chair of the Guinea-Bissau Configuration of the PBC to the Security Council [I]", 2012, en http:/ /www.un.org/en/peacebuilding/pdf/2012\%204\%20 18\% 20Chair\% 20Statement $\%$ 20SC $\%$ 20briefing $\% 2019 \% 20$ April\%202012.pdf 
Viotti, Maria L., "Statement of the Chair of the Guinea-Bissau Configuration of the PBC to the Security Council [II]", 2012, en http://www.un.org/en/peacebuilding/pdf/statement_chair_ sc_brief_gb_2012_7_26.pdf

Viотti, Maria L., "Report of the Chairpersonship of Ambassador Maria Luiza Ribeiro Viotti as Chair of the Guinea-Bissau Configuration (December 2007 - May 2013)", 2013, en http:// www.un.org/en/peacebuilding/cscs/gui/key_docs/2013\%20 6\% 203\% 20GB\% 20CSM\% 20Chair's\%20Final\% 20repor\% 20 December\%202007\%20to\%20May\%202013.pdf 